\title{
Signatures of very high energy physics in the squeezed limit of the bispectrum
}

\author{
Diego Chialva \\ Université de Mons, Service de Mécanique et gravitation, Place du Parc 20, 7000 Mons, \\ Belgium \\ diego.chialva@umons.ac.be
}

\begin{abstract}
We investigate the signatures in the squeezed limit of the primordial scalar bispectrum due to modifications of the standard theory at high energy. In particular, we consider the cases of modified dispersion relations and/or modified initial quantum state (both in the Boundary Effective Field Theory and in the New Physics Hyper-Surface formulations). Using the in-in formalism we study in details the squeezed limit of the contributions to the bispectrum from all possible cubic couplings in the effective theory of single-field inflation. We find general features such as enhancements and/or non-local shape of the non-Gaussianities, which are relevant, for example, for measurements of the halo bias and which distinguish these scenarios from the standard one (with Bunch-Davies vacuum as initial state and standard kinetic terms). We find that the signatures change according to the magnitude of the scale of new physics, and therefore several pieces of information regarding high energy physics could be obtained in case of detection of these signals, especially bounds on the scales of new physics.
\end{abstract}




\section{Contents}

$\begin{array}{llr}1 & \text { Introduction } & 1\end{array}$

2 Formalism and notation

3 Brief review of the scenarios under consideration 4

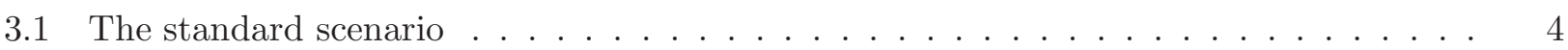

3.2 Modified dispersion relations $\ldots \ldots \ldots \ldots \ldots \ldots \ldots$

3.3 Modified initial state (BEFT and NPHS scenarios) . . . . . . . . . . . . . 6

3.4 General constraints on Bogoliubov coefficients . . . . . . . . . . . . . .

4 Bispectrum in the squeezed limit: general arguments 8

5 Bispectrum in the squeezed limit: technical analysis $\quad \mathbf{1 0}$

5.1 Example 1: Minimal coupling cubic interaction . . . . . . . . . . . . . 10

5.2 Example 2: Quartic derivative interaction . . . . . . . . . . . . . . 17

5.3 General analysis for all cubic couplings in the effective action . . . . . . . . . . 20

6 Signatures of very high energy physics in the squeezed limit 25

6.1 Enhancements . . . . . . . . . . . . . . . . . . 25

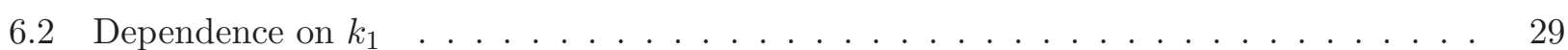

6.3 Predictions for the different modified scenarios . . . . . . . . . . . . . 30

7 Comments on the halo bias

8 Final discussion and conclusion $\quad 33$

A Appendices

A.1 Solution of the field equation in the case of modified dispersion relations . . . . . . 34

A.2 Formulas for Whightman functions and their time derivatives . . . . . . . . . . 35

A.3 Time integral in bispectrum computations with modified dispersion relations . . . . 36

\section{Introduction}

The non-Gaussian features of the primordial cosmological perturbations are going to become one of the most important experimental and theoretical tools for investigating the early history of the Universe [1. The scalar bispectrum, coming from the three-point correlator of the comoving curvature perturbation $\zeta$, is the leading contribution to the scalar non-Gaussianities in the perturbative theory.

In recent times, several reasons of interests have emerged for investigating its so-called squeezed limit, when one of the three momenta it depends on in Fourier space is much smaller than the other two: $k_{1} \ll k_{S}, k_{2,3} \simeq k_{S}$. In fact, large-scale probes of the primordial non-Gaussianities like the halo bias are expected to be soon competing with or even surpass in accuracy those of the CMBR [2 9], and this prompts for a better understanding of their sensitivity on the details of the bispectrum and the theoretical models.

Furthermore, studying the squeezed limit provides powerful results capable of distinguishing (hopefully falsify) classes of models. This is particularly true for single-field models (or more generally, for models where there are only adiabatic perturbations). All single-field models (adiabatic perturbations) with Bunch-Davies vacuum and standard kinetic terms (we call this the standard scenario from now on) predict the so-called local form of the bispectrum and a very low level of non-Gaussianities in 
the limit [10 12]. Remarkably, measurements of the halo bias could discriminate cases with different underlying assumptions [13, 14]: for example models with modified kinetic terms whose bispectrum template is maximized on equilateral configurations, or with initial state different than the BunchDavies, in which case the proposed template is maximized on folded configurations [15].

In this paper, we want to look at the field theory cosmological predictions for the squeezed limit of the bispectrum for single-field slow-roll inflationary models, investigating the effects of two very simple modifications of the standard scenario:

1) the presence of higher derivative corrections to the action that are unsuppressed at a certain physical scale $\Lambda$ and modify the dispersion relations of the perturbation fields [16 19$]$,

2) the choice of an initial state other than the Bunch-Davies vacuum2, reflecting the effects of different physics at higher energies/earlier times as proposed in 32 37] (we consider both the BEFT and the NPHS scenarios, see section 3.3 for a brief review).

There are in fact several reasons, see section 4 , to expect that in such scenarios the standard result for the squeezed limit of the bispectrum will not occur.

The description of the physics at high energies is largely unknown, and, at the same time, there are various different models of single-field inflation. It is therefore important to derive general results, as indeed was in the spirit of [11,12. We will therefore deal with this topic adopting the same phenomenological approach and scenarios of [15, 20, 38, not assuming specific detailed high-energy models, but still obtaining quantitative as well as qualitative results. We will present in more details the approach and the scenarios in section 3 . It is also important to stress that we consider the realistic squeezed limit, where $k_{1}$ is small but yet nonzero, as defined in [12 14], in view of possible future observations. Finally, we study the contribution from all possible cubic couplings in the effective action for single-field inflation à-la Weinberg [39].

Beside [15,38] (which studied the bispectrum, but not in the squeezed limit), modified initial states have been proposed and studied in [32,37,40,41] and many successive papers. The scenarios that we consider are well-defined phenomenologically and formally, as investigated by a large number of works, see for example [40,41]. Modified initial conditions for the perturbations can derive for example from the preceding evolution of the universe (see the examples of [42,43]) or from integrating out heavy fields [44, 45]. On the other hand, modified dispersion relations occur in a variety of models, such as certain realization of quantum gravity, like the Hořava-Lifshitz model [46], or also in braneworld models [47 51], and are of interest for the studies of Lorentz violation [52,53]. They occur as well in the effective theory when the fields propagate with small speed of sound [54,55].

The outline of the paper is as follows: we begin in section 2 with a very brief presentation of the necessary formalism, followed by a short review of the scenarios we consider in section 3, We then study the bispectrum in the squeezed limit. First, we provide a general discussion of the (expected) results and their physical interpretation in section 4 .

\footnotetext{
${ }^{1}$ As in [20, our scenario differs from that considered in [21,22], where the only modification to the field equations was a change in the speed of sound (the modifications at the level of the Lagrangian depended only on first order derivatives of the fields). In particular, we include also derivatives of higher order, and the effect on the field equations is more profound. In many scenarios with time-varying speed of sound the Maldacena's condition [10, 11] on the squeezed limit of the bispectrum holds, see [12,23,25]. Similarly, see [12], in the adiabatic ekpyrosis scenario of [26, 27]. Some contrived model (non-attractor background) 28] do not satisfy the relation [12.

${ }^{2}$ The halo bias and the squeezed limit for this case have been studied in the literature mostly by using the template of 15 and not the actual field theoretical results for the bispectrum. We will show that this appreciably changes the outcomes (this was noted also in [29], which however still employed the template and did not study in details the field theoretical results). After the appearance of this article (August 2011), we were signalled reference [30], which also makes remarks, focusing on the presence of enhancements, about the squeezed limit in a particular formalism (density matrix approach) with a specific initial condition for the fields (akin to the one called BEFT in section 3.3 which explicitly breaks scale invariance) and dealing only with one specific cubic coupling (the interaction (18)). See also [31] which analyses the same scenario. Also in these articles the study of the general field theoretical results was not undertaken and part of the signatures were missed (see our sections 5 and 6 ).
} 
We then present the technical computation of the bispectrum in the squeezed limit in section 5 , For the reader's advantage, we proceed in three steps. First, in sections 5.1 and 5.2, we discuss in full details two examples of contributions from two well-known cubic coupling (with and without higherderivatives). These example are meant to help the reader to quickly grasp the features of the more general computations and results later on.

Second, in section 5.3, we move to the general analysis and study the contributions to the bispectrum in the squeezed limit from all allowed couplings in the effective theory (single-field).

As a third step, armed with these general results, in section 6 we analyse the features of the various contributions, individuating the leading ones and providing the predictions for the bispectrum in the squeezed limit for the modified scenarios we have considered.

Finally, we briefly comment on the consequences of our results on the halo bias in section 7 , and conclude in 8 . Some useful equations and material, which would have made heavy the main text, have been moved to the appendices.

\section{Formalism and notation}

Let us first introduce the basic notation and elements of the formalism of cosmological perturbation theory, for more details see [10]. Scalar perturbations in single-field slow-roll inflationary models are efficiently parametrized by the comoving curvature perturbation 3

$$
\zeta(\eta, \vec{x})=\int \frac{d^{3} k}{(2 \pi)^{\frac{3}{2}}} \zeta_{\vec{k}}(\eta) e^{i \vec{k} \cdot \vec{x}}
$$

The slow-roll parameters are defined as $\epsilon=\frac{\dot{\phi}^{2}}{2 H^{2} M_{\text {Planck }}^{2}}, \eta_{\text {sl }}=-\frac{\ddot{\phi}}{\dot{\phi} H}+\frac{\dot{\phi}^{2}}{2 H^{2} M_{\text {Planck }}^{2}}$, where $H$ is the Hubble rate. $\phi$ is the inflaton (background), and $a(\eta) \sim-\frac{1}{H \eta}(\eta<0)$ is the scale factor at zeroth order in slow-roll. Throughout the paper, dots (primes) indicate derivatives with respect to cosmic time $t$ (conformal time $\eta$ ).

The two-point function is defined as

$$
\left\langle\zeta\left(\vec{k}_{1}\right) \zeta\left(\vec{k}_{2}\right)\right\rangle=(2 \pi)^{3} \delta^{(3)}\left(\vec{k}_{1}+\vec{k}_{2}\right) P\left(k_{1}\right), \quad k_{1}=\left|\vec{k}_{1}\right| .
$$

and, following [56], we define the bispectrum $B$ as

$$
\left\langle\zeta_{\vec{k}_{1}}(\eta) \zeta_{\vec{k}_{2}}(\eta) \zeta_{\vec{k}_{3}}(\eta)\right\rangle=(2 \pi)^{3} \delta^{(3)}\left(\sum_{i} \vec{k}_{i}\right) B\left(\vec{k}_{1}, \vec{k}_{2}, \vec{k}_{3}, \eta\right)
$$

where, at leading order in the perturbative expansion,

$$
\left\langle\zeta\left(\eta, \vec{x}_{1}\right) \zeta\left(\eta, \vec{x}_{2}\right) \zeta\left(\eta, \vec{x}_{3}\right)\right\rangle=-2 \operatorname{Re}\left(\int_{\eta_{\text {in }}}^{\eta} d \eta^{\prime} i\left\langle\psi_{\text {in }}\left|\zeta\left(\eta, \vec{x}_{1}\right) \zeta\left(\eta, \vec{x}_{2}\right) \zeta\left(\eta, \vec{x}_{3}\right) H_{(I)}\left(\eta^{\prime}\right)\right| \psi_{\text {in }}\right\rangle\right) .
$$

Here, $\eta_{\text {in }},\left|\psi_{\text {in }}\right\rangle$ are the initial time and state, and $H_{(I)}$ is the interaction Hamiltonian.

A key ingredient in the computation of correlators is the Whightman function

$$
(2 \pi)^{3} \delta^{(3)}\left(\vec{k}_{1}+\vec{k}_{2}\right) G_{\vec{k}}\left(\eta, \eta^{\prime}\right) \equiv\left\langle\zeta_{\vec{k}_{1}}(\eta) \zeta_{\vec{k}_{2}}\left(\eta^{\prime}\right)\right\rangle=(2 \pi)^{3} \delta^{(3)}\left(\vec{k}_{1}+\vec{k}_{2}\right) \frac{H^{2}}{\dot{\phi}^{2}} \frac{f_{k}(\eta)}{a(\eta)} \frac{f_{k}^{*}\left(\eta^{\prime}\right)}{a\left(\eta^{\prime}\right)}
$$

where $f_{\vec{k}}(\eta), f_{\vec{k}}^{*}(\eta)$ are two linearly independent solutions of the field equation

$$
f_{\vec{k}}^{\prime \prime}+\left(\omega(\eta, \vec{k})^{2}-\frac{z^{\prime \prime}}{z}\right) f_{\vec{k}}=0 \quad z=\frac{a \dot{\phi}}{H}
$$

\footnotetext{
${ }^{3}$ We follow the conventions in [10, 15],20, 38; ; other authors call this perturbation $\mathcal{R}$ to distinguish it from the uniform energy-density curvature perturbation.
} 
such that, once quantized,

$$
\zeta_{\vec{k}}(\eta)=\frac{f_{\vec{k}}(\eta)}{z} \hat{a}_{\vec{k}}^{\dagger}+\frac{f_{\vec{k}}^{*}(\eta)}{z} \hat{a}_{\vec{k}}
$$

Imposing the standard commutation relation on the operators $\hat{a}_{\vec{k}}^{\dagger}, \hat{a}_{\vec{k}}$, entails a certain normalization for the Wronskian of $f_{\vec{k}}^{*}(\eta), f_{\vec{k}}(\eta)$. Different choices for $f_{\vec{k}}^{*}(\eta), f_{\vec{k}}(\eta)$ correspond to different choices of initial state [10]. In equation (6),$\omega$ is the (comoving) frequency as read from the effective action: we will limit ourselves to the isotropic case, where $\omega$ depends only on $k \equiv|\vec{k}|$, dropping the arrow symbol.

\section{Brief review of the scenarios under consideration}

To ease the discussion of the results for the three-point function and make the paper self-contained, let us recap here the main features of the single-field scenarios we will deal with. For more details see $[15,20,32,38$.

\subsection{The standard scenario}

In the standard scenario, as defined in the introduction, the kinetic terms are standard, the dispersion relation is the usual Lorentzian one and the mode functions $f_{\vec{k}}(\eta)$ are determined by choosing the Bunch-Davies vacuum, so that

$$
\omega(\eta, k)^{2}=k^{2}, \quad f_{k}(\eta)=\frac{\sqrt{\pi}}{2} e^{i \frac{\pi}{2} \nu+i \frac{\pi}{4}} \sqrt{-\eta} H_{\nu}^{(1)}(-k \eta),
$$

where $H^{(1)}$ is the first Hankel's functions with $\nu=\frac{3}{2}+\frac{1-n_{s}}{2}$, and the equations and solutions are extrapolated up to $\eta_{\text {in }}=-\infty$ where the initial state has been chosen as the empty adiabatic vacuum (Bunch-Davies). At leading order in slow-roll the spectral index reads $n_{s}=1-6 \epsilon+2 \eta_{\mathrm{sl}}$ and the late time behaviour of the two-point function is

$$
P_{\mathrm{st}}(k) \underset{\eta \rightarrow 0}{\sim} \frac{H^{2}}{4 M_{\text {Planck }}^{2} \epsilon k^{3}}
$$

where the suffix st indicates that we are considering the standard case.

\subsection{Modified dispersion relations}

Higher-derivative corrections to the action are deeply rooted in the well-established effective field theory framework. In fact, one expects that at certain times at least some of the higher derivative corrections to the action cannot be treated perturbatively, because, since the metric scale factor $a(\eta)$ is rapidly decreasing back in time, the suppressing factors, given by powers of ratios such as $\frac{p}{\Lambda}=\frac{k}{a(\eta) \Lambda}$, where $\Lambda$ is the relevant high-energy scale, are not small any more. These no longer small corrections can lead to modified dispersion relations. As recalled in the introduction, such possibility appears in a variety of scenarios, see for example those in [46 55].

As we said, we adopt a phenomenological approach and consider generic modifications to the dispersion relation due to higher-derivative corrections to the effective action, encoding them in a function $F\left(-\frac{p}{\Lambda}\right)$ by writing the comoving frequency as

$$
\omega(\eta, k)=a(\eta) \omega_{\text {phys }}(p)=a(\eta) p F\left(-\frac{p}{\Lambda}\right)=k F\left(\frac{H}{\Lambda} k \eta\right), \quad F(x \rightarrow 0) \rightarrow 1, \quad \frac{H}{\Lambda} \ll 1 .
$$

In [20,57, it has been shown that if the modified dispersion relations always satisfy the WKB conditions (adiabaticity) at early times (that is, WKB is violated only when the dispersion is in the 


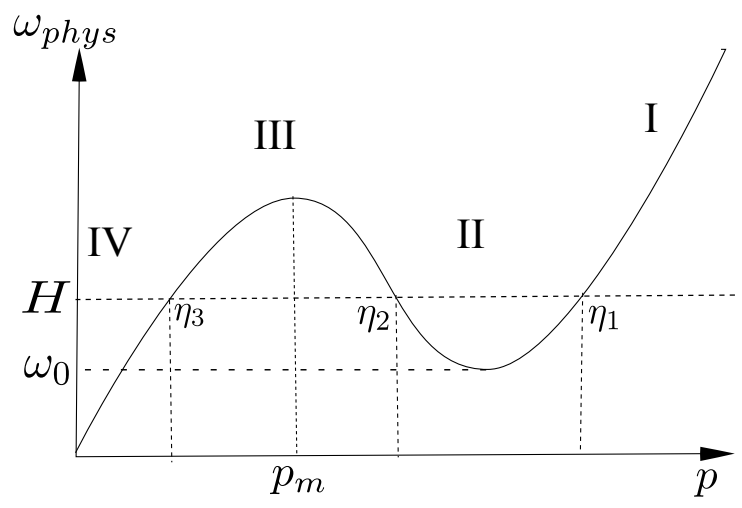

Figure 1: Generic example of dispersion relation with violation of WKB at early times.

standard linear regime $\omega \simeq k$ as usual) the bispectrum is similar to that of the standard scenario, and so it will be its squeezed limit. This is easily understood, since the particle production is practically absent in those cases.

Instead, 20] has shown that the bispectrum is particularly sensitive to modifications that lead to a violation of the WKB conditions (adiabaticity) at early times, where particle production is more substantial. We will therefore focus on those scenarios.

The generic shape of a dispersion relation with WKB violation once at early times is depicted in figure1. We stress that we are not proposing any specific model, which would be a strong assumption on the high energy physics: we consider the most generic function $F\left(-\frac{p}{\Lambda}\right)$ in equation (10), with the only constraint that adiabaticity is once violated at early times (it is easy to extend our analysis to multiple violations). We are in fact interested in the general consequences on the bispectrum from this scenario. Similar phenomenological approach and generic dispersion relations have been considered and discussed, studying the spectrum, for example in the references [16 19, see also [52,53].

A general treatment of the field equation (6) in these cases yields the following solution [20]:

$$
f_{k}(\eta)= \begin{cases}\varsigma_{k} u_{1}(\eta, k) & \mathrm{I}: \eta<\eta_{\mathrm{I}}^{(k)} \\ B_{1} \mathcal{U}_{1}(\eta, k)+B_{2} \mathcal{U}_{2}(\eta, k) & \mathrm{II}: \eta_{\mathrm{I}}^{(k)}<\eta<\eta_{\mathrm{I}}^{(k)} \\ \alpha_{k}^{\mathrm{mdr}} u_{1}(\eta, k)+\beta_{k}^{\mathrm{mdr}} u_{2}(\eta, k) & \mathrm{III}: \eta_{\mathrm{II}}^{(k)}<\eta<\eta_{\mathrm{II}}^{(k)} \\ D_{1} \mathcal{V}_{1}(\eta, k)+D_{2} \mathcal{V}_{2}(\eta, k) & \mathrm{IV}: \eta_{\mathrm{II}}^{(k)}<\eta\end{cases}
$$

where $u_{1,2}(\eta, k)$ can be very well approximated using the WKB method, while $\mathcal{V}_{1,2}(\eta, k)$ and $\mathcal{U}_{1,2}(\eta, k)$ need to be found by other means, as the WKB conditions are not satisfied 4 .

As known, given equation (6), the times $\eta_{\mathrm{I}, \mathrm{II}, \mathrm{II}}^{(k)}$ where the WKB approximation first fails are in proximity of the turning points of $V(\eta, k)^{2} \equiv \omega(\eta, k)^{2}-\frac{z^{\prime \prime}}{z}$, see [58, 59]. They depend on $k$. When clear from the context we will omit the label ${ }^{(k)}$. Looking at figure 1, these times will be in proximity of $\eta_{1,2,3}$ [16 20]. The coefficients $D_{1,2}, B_{1,2}, \alpha_{k}^{\mathrm{mdr}}, \beta_{k}^{\mathrm{mdr}}$ are obtained by asking for the continuity of the function and its first derivative, and by imposing the Wronskian condition $\mathcal{W}\left\{f, f^{*}\right\}=-i$ in order to have the standard commutation relations in the quantum theory. The label "mdr" indicates that we are considering the scenario of modified dispersion relations.

The error made by using these approximations can be made small at will by going to higher order in the approximation, see for example [20] or [59]. The techniques are standard in cosmology and allow controlled global approximations of the equations solutions and/or Green functions.

\footnotetext{
${ }^{4}$ Some different general approximations are possible in this case, see 20] and the appendix A.1 With particular choices of time coordinate, also the WKB approximation can still be applied sometimes, see 58.
} 
The details of the solution can be found in 20, and are briefly reviewed in the appendix A.1, Some of the salient features needed here are that:

- backreaction constraints the interval $\left[\eta_{\mathrm{I}}, \eta_{\mathrm{I}}\right]$ of WKB violation to be very small, that is

$$
\Delta=\frac{\eta_{\mathrm{I}}-\eta_{\mathrm{II}}}{\eta_{\mathrm{I}}} \ll 1
$$

- expanding for small $\Delta$, we obtain generically (i.e., independently of the detailed form of $\omega(\eta, k)$ )

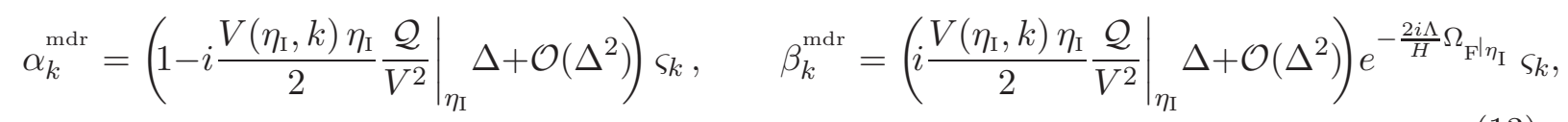

where $V(\eta, k)^{2} \equiv \omega(\eta, k)^{2}-\frac{z^{\prime \prime}}{z}$. Here, $\left.\frac{\mathcal{Q}}{V^{2}}\right|_{\eta_{\mathrm{I}}}$ is the order 1 factor signalling the violation of the WKB conditions at $\eta_{\mathrm{I}}$, and $\mathcal{Q}$ is reported in equation (105) in appendix A.1. The magnitude of $\left|\beta_{k}^{\mathrm{mdr}}\right|$ is constrained by backreaction. We will discuss more of the $k$-dependence and the magnitude of these coefficients in section 3.4 .

- we choose $\varsigma_{k}=1$ picking up the usual adiabatic vacuum.

At late time the two-point function does not differ much from the standard one, since the Bogoliubov coefficient is quite constrained by backreaction (see section 3.4). This is good for the agreement with the observations on the (late time) spectrum. At leading order in $\epsilon, \beta_{k}^{\mathrm{mdr}}, \Delta$,

$$
P_{\text {mdr }}(k) \underset{\eta \rightarrow 0}{\sim} \frac{H^{2}}{4 M_{\text {Planck }}^{2} \epsilon k^{3}}\left(1+2 \operatorname{Re}\left(\beta_{k}^{\text {mdr }}\right)\right) .
$$

In the case of the bispectrum it will be important the behaviour of the Whightman function at earlier times, which shows more relevant modifications, see appendix A.2.

\subsection{Modified initial state (BEFT and NPHS scenarios)}

Let us review the two implementations of the modified initial state approach that have been first proposed in 32 37, reflecting the effects of the physics at higher energies/earlier times than inflation in setting the initial/boundary condition for the perturbation fields.

- BEFT approach: one follows an effective theory approach by fixing the boundary conditions for the fields at the finite time $\eta_{c}$ (beginning of inflation) independently of the modes $k$, through a Boundary Effective Field Theory (BEFT), which accounts for the high energy physics via renormalization 35 37. This kind of boundary condition strongly breaks scale invariance, being imposed for all modes $k$ at the same boundary time. The (cutoff) scale $\Lambda$, proper of the effective action formulation, is not related to the boundary time $\eta_{c} 3537$.

- NPHS approach: consider the effective theory valid up to a certain energy scale $\Lambda$, and choose the boundary conditions for the solution to the field equation at the New Physics Hyper-Surface (NPHS) corresponding to when the physical momentum reaches that cutoff scale [32 34]. In this case, the cutoff is imposed in a scale-invariant way via the condition $\frac{k}{a\left(\eta_{c}\right)}=\Lambda$, so that the time $\eta_{c}$ when the initial state is picked is $k$ - and $\Lambda$ - dependent. At zero slow-roll order, $\eta_{c}=-\frac{\Lambda}{k H}$.

To spare notation, we use the symbol $\eta_{c}$ for both the NPHS and BEFT cases and make clear in the context which approach we are following. In both cases, we stress that the initial condition is fixed when the modes $k$ are well within the horizon, that is, for $\eta_{c}$ 's such that $\left|k \eta_{c}\right| \gg 1$. 
The physical motivation why such modified initial states can be chosen is the fact that inflation and cosmological perturbation theory are effective theories valid below a certain energy scale (if the theory was valid at all scales, the only sensible choice would be the so-called Bunch-Davies vacuum) [40,41. Many studies have proven that these initial states and the perturbation theory defined upon them are well-defined also in the formal sense (for example, see [40,41]). As in [15, 38, when studying these scenarios we adopt a phenomenological approach.

A modified initial condition may present both a Gaussian part and an intrinsic non-Gaussian one. As we will discuss in section 4, using the result of [60,61 - see also the comment in [15] - one finds that the leading corrections to the bispectrum in the squeezed limit due to intrinsic non-Gaussianities of the initial condition, if present, would be in line with the standard result, with a local form and a suppressed amplitude, because of backreaction and lack of cumulation with time.

The Gaussian part of the initial condition, which modifies the Whightman functions of the perturbation theory, will instead turn out to be responsible for new and more dominant features of the squeezed limit. As for this part, both in the BEFT and NPHS cases the solution of the field equation is of the Bogoliubov form [15, 32, 38]:

$$
f_{k}(\eta)=\alpha_{k}^{\mathrm{mis}} \sqrt{-\eta} H_{\nu}^{(1)}(-k \eta)+\beta_{k}^{\mathrm{mis}} \sqrt{-\eta} H_{\nu}^{(2)}(-k \eta),
$$

where $\alpha_{k}^{\text {mis }}, \beta_{k}^{\text {mis }}$ are determined by the specific boundary conditions imposed on the solution at the time $\eta_{c}$, and by the Wronskian condition, which translates into $\left|\alpha_{k}^{\text {mis }}\right|^{2}-\left|\beta_{k}^{\text {mis }}\right|^{2}=1$. The Bogoliubov coefficients depend on the mode $k$, the cutoff scale and the time $\eta_{c}$ (these latter are related in the NPHS case, but not in the BEFT, as we said). The label "mis" indicates that we are dealing with the case of modified initial state.

At late time the two-point function does not differ much from the standard one, because of the smallness of $\beta_{k}^{\text {mis }}$ due to backreaction constraints (see section 3.4). At leading order in slow-roll and $\beta_{k}^{\text {mis }}$

$$
P_{\text {mis }}(k) \underset{\eta \rightarrow 0}{\sim} \frac{H^{2}}{4 M_{\text {Planck }}^{2} \epsilon k^{3}}\left(1+2 \operatorname{Re}\left(\beta_{k}^{\text {mis }} e^{-i \operatorname{Arg}\left(\alpha_{k}^{\text {mis }}\right)}\right)\right) .
$$

Once again, for the bispectrum it will be important the behaviour of the Whightman function at earlier times, which shows more relevant differences, see appendix A.2.

\subsection{General constraints on Bogoliubov coefficients}

The magnitude and scale $(k)$ dependence of the coefficients $\beta_{k}^{\mathrm{mdr}}, \beta_{k}^{\mathrm{mis}}$ entering respectively equations (14) and (16) are determined in full details by the specific model or boundary condition giving rise to the modified dispersion relation or modified initial state. However, to be in accordance with observations and to avoid backreaction stopping the slow-roll inflationary evolution, these coefficients are subject to a series of phenomenological constraints, see for example [20, 38]:

- the observations on the spectral index show that $-k \frac{d \log \left(k^{3} P(k)\right)}{d k}$ is small, so that $\beta_{k}^{\mathrm{mdr}}, \beta_{k}^{\mathrm{mis}}$ must be slowly varying with $k$ at the observed scales;

- backreaction imposes two further constraints:

- the total energy density must be finite. In general this demands that $\left|\beta_{k}^{\text {mdr }}\right|,\left|\beta_{k}^{\text {mis }}\right|$ decay faster than $k^{-2}$ at large $k$;

- preserving the slow-roll inflationary evolution enforces the constraint [20, 38]

$$
\left|\beta_{k}^{\mathrm{mdr}}\right|,\left|\beta_{k}^{\mathrm{mis}}\right| \leq \sqrt{\epsilon|\mu|} \frac{H M_{\mathrm{Planck}}}{\Lambda^{2}}, \quad \mu \equiv \eta_{\mathrm{sl}}-\epsilon .
$$


In the following we will keep indicating the scale dependence of these coefficients with the label $k$. We will also continue distinguishing the modified initial state scenario from that with modified dispersion relations using the labels "mis" and "mdr".

\section{Bispectrum in the squeezed limit: general arguments}

We will now discuss the three-point function. Before presenting the detailed analysis, it is useful to outline the points that make the difference with the standard scenario in the cases we consider.

To make the section self-contained, let us first recall briefly that the scalar bispectrum is a threepoint correlator for the scalar perturbation field evaluated at a late time after horizon exit of the observed modes. It is calculated on an initial state defined at an initial (conformal) time $\eta_{\text {in }}$, which is then evolved up to the time the bispectrum is evaluated at, and back to $\eta_{\text {in }}$ (in-in formalism, we are using the interaction picture). Its leading perturbative formula has been presented in equation (4).

The standard result for the squeezed limit $k_{1} \ll k_{2,3} \sim k_{S}$ follows from very simple arguments [10 12]:

a) for an initial Bunch-Davies vacuum state and a standard comoving Lorentzian dispersion relation the non-Gaussianities are essentially generated at horizon exit, and thus the most relevant part of the time evolution is from horizon exit of the modes until the time the bispectrum is evaluated at;

b) since $k_{1} \ll k_{2,3}$ in the squeezed limit, the horizon-exit time for the perturbations depending on $k_{2,3}$ occurs much later than the one for the perturbation depending on $k_{1}$. The latter then acts as a background for the other perturbations, shifting their horizon-exit time. This leads to a certain dependence of the result on $k_{1}$ (dubbed "local") and on the spectral index (which is related to the shift of horizon-exit time). It is called the Maldacena's consistency relation [10 12].

These pieces of physical information are encoded in the form of the Whightman functions (the twopoint functions, see section (2) of the standard scenario. Every higher-order correlator is written in terms of them because of Wick's theorem. In particular, their form is such that the time integral from $\eta_{\text {in }}$ to $\eta \sim 0$ coming from the time evolution in the bispectrum formula (4) is dominated by the upper limit of integration (see sections 5, 5.1.1). This is how condition a) is encoded mathematically.

It is therefore natural to expect that if the Whightman functions are modified, in particular in a way such that the time-integral in the bispectrum formula picks up other important contributions violating conditions $a$ ) and/or $b$ ), then the squeezed limit will be different from the standard one. This is what occurs in the scenarios of modified dispersion relation and of modified initial state/condition.

At this point one could think that if the scenarios allow modifications to the Whightman functions, one would completely loose general predictivity on the squeezed limit. However, we will show that this is not the case: new general physical arguments based on the concepts of "particle creation/content" 5 , interference and accumulation in time, take the place of those operating in the standard scenario.

'Particles' arise in different ways in the two scenarios we consider, see section 3 . In the case of modified initial state the initial condition for the field mode functions is fixed at $\eta_{\text {in }}$ when the modes are well within the horizon (adiabaticity is satisfied), but the physics at times/scales preceding $\eta_{\text {in }}$ can be parametrized and interpreted in terms of what we will call the 'particle content' of the state. The idea is that the physics at scale higher than inflation has generated an initial state that is not the adiabatic vacuum, but an excited one [42 45] - hence its nonzero energy and particle content. In the case of modified dispersion relation, instead, the time evolution of the dispersion relation can lead to 'particle production' even if the initial state is the standard empty adiabatic vacuum [20,57].

\footnotetext{
${ }^{5}$ As well-known, the concept of particle is not well-defined on time evolving background. Approximate concepts with respect to comoving observers have a standard use in connection with adiabaticity, see 62 67. Recall that adiabaticity in this context concerns the time evolution of certain quantities (usually the effective frequency/mass, from the quadratic part of the action [62 67]) and is independent and conceptually different from non-Gaussianity.
} 
It is the presence of this early energy and 'particle' content that generates the additional nonGaussianities. Of course, this content is severely constrained by backreaction on inflation, as we will review.

The effects of particle content/creation will be stronger at early times (before dilution by cosmic expansion). The fact that the time-evolution integral extends to such early times explains why the bispectrum (and in principle all higher-order correlators) are particularly sensitive to these modifications to the Whightman functions. Indeed, the spectrum is affected by them as well, but much less so, because it does not involve an integration over time and, especially for observational reasons, it is computed at late times, after the horizon exit of the perturbation, where the modifications are negligible.

The modifications of the Whightman functions will affect all correlators and lead to different results, compared to the standard case, even for the simplest couplings. The new features in the squeezed limit will concern scale dependence as well as magnitude (enhancements). Indeed, although particle creation is certainly strongly constrained by backreaction, it can lead to interference and phase cancellation in the integrand of the time integral in the bispectrum, giving rise to enhancements.

As we will see, the greatest effect occurs when the largest contributions to the oscillating phase of the integrand cancel out so that the suppression due to oscillations is strongly reduced. This happens when the early particle content for the perturbations depending on the largest modes $k_{2,3}$ is relevant and interference occurs among them. At those times, the perturbation depending on $k_{1} \ll k_{S}$, initially subhorizon, may or may not be superhorizon yet. As we will see, this condition will depend on the magnitude of $k_{1}, k_{S}$ and the scale of the new physics. There can then occur two different cases.

If the perturbation depending on $k_{1}$ is not already superhorizon at those times, we do not expect (and do not get indeed) the same $k_{1}$-dependence (local shape) as in the standard result, because that is entirely determined by the superhorizon condition.

If instead the $k_{1}$-perturbation was already superhorizon, we obtain a local shape and an effect due to the shift of the horizon crossing time for the other perturbations as in the standard scenario. However, also in this case we have a new result, because the overall amplitude of the bispectrum does not match the standard one, and can also be enhanced. This is a consequence of the additional nonGaussianities generated by the particle content/creation at early times for the perturbations depending on $k_{2,3}$, see sections 5 and 6 .

In the case of modified initial conditions, there can also be non-Gaussianities intrinsic to the initial condition. However, their effects are subdominant with respect to those due to the modifications of the Whightman functions. In fact, in the squeezed limit the contribution of intrinsic non-Gaussianities is in line with the standard one: local form and very suppressed amplitude (because of backreaction).

This can be seen in various ways. First of all, the general results for the leading contributions to the bispectrum from intrinsic non-Gaussianities of the initial conditions were calculated in [60, 61, using the BEFT formalism - see also the comment in [15]-. By taking the squeezed limit of those results one obtains an outcome in line with the standard one. One can also argue that the contribution of non-Gaussianities intrinsic to the initial condition is negligible from general arguments. Indeed, those non-Gaussianities, already strongly constrained by backreaction, are nonzero only at the initial time. Thus, their contributions lack the integration over time. This makes them subdominant with respect to the contributions due to the modified Whigthman functions (Gaussian part), where interference and time accumulation occur and enhance the result, as we will see.

Generally predictable features of the squeezed limit in the modified scenarios indeed appear precisely because the most important new effects are played by the modifications of the Whightman functions and not by specific couplings or peculiar initial intrinsic non-Gaussianities. The results are then dominated (we will see in what measure) by the general features of particle content/creation, interference, accumulation and sub-/superhorizon evolution. This permits to constrain and possibly falsify entire classes of models as the differences between the specific single-field slow-roll models enter 
the subleading corrections.

We are now going to investigate these features of the squeezed limit of the bispectrum by performing the analysis at the rigorous level of the field theory description using the in-in formalism. We perform a thorough analysis from this point of view, providing general result for all single-field models of inflation within the scenarios of section 3, and studying all cubic couplings that arise in an effective theory formalism à-la Weinberg [39].

In the case of modified initial state, we will also show that the field theoretic result for the squeezed limit is very different from those obtained in previous studies, see [13, 14, which used the folded template proposed for CMBR analysis in [15]. This disagreement could have been anticipated, since the standard evaluators (cosine and fudge factor) for the matching between the template [15] and theoretical prediction [38] indicate that the two depart more and more for large $k_{L} \eta_{c}$, where $k_{L}$ is the largest momentum and $\eta_{c}$ is the time when the boundary condition picking up the initial state is imposed, see [15]. Even more importantly, the template does not depend on the scale $\eta_{c}$, and therefore taking the squeezed limit in the full result is different than taking it in the template, because of the presence of distinct scales.

\section{Bispectrum in the squeezed limit: technical analysis}

We present here the most technical part of the paper, where we calculate the contributions to the squeezed limit of the bispectrum. We subdivide our presentation in three parts.

- The first two parts (sections 5.1 and 5.2) consist of two detailed examples (for cubic couplings with and without higher derivatives), to illustrate in details what are the differences between the scenarios we discuss and the standard one.

Let us stress that at this point we are not setting apart the interactions in these examples as special or dominant compared to all the other possible ones. We choose them simply because they are two well-known and well-studied cubic interactions, see [10,15, 20, 38, 68, and thus the discussion should be easier to follow for the reader. Only after the general analysis has been performed in section 5.3 we will come back to the question whether these, or other, interactions play a predominant role (see section 6).

- In section 5.3 we then deal with the full general analysis of the squeezed limit of the bispectrum, considering all possible cubic interactions in the effective action for the inflaton.

The results we obtain will be then fully analysed and discussed in section 6. We will find out what are the leading features of the bispectrum in the squeezed limit for the modified scenarios and what are the prediction for each specific modified scenario.

\subsection{Example 1: Minimal coupling cubic interaction}

We begin by studying the example of the cubic interaction [10]:

$$
H_{(I)}=-\int d^{3} x a^{3}\left(\frac{\dot{\phi}}{H}\right)^{4} \frac{H}{M_{\text {Planck }}^{2}} \zeta_{c}^{\prime 2} \partial^{-2} \zeta_{c}^{\prime} .
$$

We call this interaction the "minimal coupling cubic interaction" because it is already present in the simplest case of a scalar field (inflaton) minimally coupled to gravity [10].

\footnotetext{
${ }^{6}$ However, the squeezed limit of their contributions to the bispectrum has not been studied in the modified scenarios we consider.
} 
We have followed the practice of [10] writing this interaction in terms of the field redefinition 7

$$
\zeta=\zeta_{c}+\frac{1}{8} \frac{\dot{\phi}^{2}}{H^{2} M_{\text {Planck }}^{2}} \zeta_{c}^{2}+\frac{1}{4} \frac{\dot{\phi}^{2}}{H^{2} M_{\text {Planck }}^{2}} \partial^{-2}\left(\zeta_{c} \partial^{2} \zeta_{c}\right)+\frac{1}{2} \frac{\ddot{\phi}}{\dot{\phi} H} \zeta_{c}^{2} .
$$

The two-point function and the quadratic part of the action are the same for $\zeta$ and $\zeta_{c}$ [10].

Using the definitions of the slow-roll parameters, the bispectrum of $\zeta$, which is the relevant one for observations, is related to the three-point function for $\zeta_{c}$ as

$$
\begin{aligned}
\left\langle\zeta_{\vec{k}_{1}}(\eta) \zeta_{\vec{k}_{2}}(\eta) \zeta_{\vec{k}_{3}}(\eta)\right\rangle=\left\langle\zeta_{c, \vec{k}_{1}}(\eta) \zeta_{c, \vec{k}_{2}}(\eta) \zeta_{c, \vec{k}_{3}}(\eta)\right\rangle & \\
& +(2 \pi)^{3} \delta^{(3)}\left(\sum_{i} \vec{k}_{i}\right) \sum_{\substack{i=1 \\
\bmod 3}}^{3}\left(\epsilon\left(\frac{3}{2}+\frac{k_{i+1}^{2}+k_{i+2}^{2}}{2 k_{i}^{2}}\right)-\eta_{\mathrm{sl}}\right) P\left(k_{i+1}\right) P\left(k_{i+2}\right),
\end{aligned}
$$

where

$$
\left\langle\zeta_{c}\left(\eta, \vec{x}_{1}\right) \zeta_{c}\left(\eta, \vec{x}_{2}\right) \zeta_{c}\left(\eta, \vec{x}_{3}\right)\right\rangle=-2 \operatorname{Re}\left(\int_{\eta_{\text {in }}}^{\eta} d \eta^{\prime} i\left\langle\psi_{\text {in }}\left|\zeta_{c}\left(\eta, \vec{x}_{1}\right) \zeta_{c}\left(\eta, \vec{x}_{2}\right) \zeta_{c}\left(\eta, \vec{x}_{3}\right) H_{(I)}\left(\eta^{\prime}\right)\right| \psi_{\text {in }}\right\rangle\right),
$$

and where $\eta \sim 0$ is a late time when all modes $k_{i}$ are outside the horizon.

As we know from the review in sections $3.2,3.3$ the modifications affecting the second line of (20) via the spectra $P\left(k_{i}\right)$ 's for the modified scenarios at late time are very suppressed. Hence, we focus on the connected contribution (21). From (18), (21)

$$
\left\langle\zeta_{c, \vec{k}_{1}}(\eta) \zeta_{c, \vec{k}_{2}}(\eta) \zeta_{c, \vec{k}_{3}}(\eta)\right\rangle=2 \operatorname{Re}\left(-i(2 \pi)^{3} \delta^{(3)}\left(\sum_{i} \vec{k}_{i}\right)\left(\frac{\dot{\phi}}{H}\right)^{4} \frac{H}{M_{\text {Planck }}^{2}} \int_{\eta_{\text {in }}}^{\eta} d \eta^{\prime} \frac{a\left(\eta^{\prime}\right)^{3}}{k_{3}^{2}} \prod_{i=1}^{3} \partial_{\eta^{\prime}} G_{k_{i}}\left(\eta, \eta^{\prime}\right)+\text { permutations }\right)
$$

We will now present the results for the standard and modified scenarios. Our notation is as follows. We will write the result for the three-point function in powers of $\left|\beta_{k_{i}}\right|$. Up to linear order,

$$
\left\langle\zeta_{c, \vec{k}_{1}} \zeta_{c, \vec{k}_{2}} \zeta_{c, \vec{k}_{3}}\right\rangle=\delta_{0}\left\langle\zeta_{c, \vec{k}_{1}} \zeta_{c, \vec{k}_{2}} \zeta_{c, \vec{k}_{3}}\right\rangle+\delta_{\beta}\left\langle\zeta_{c, \vec{k}_{1}} \zeta_{c, \vec{k}_{2}} \zeta_{c, \vec{k}_{3}}\right\rangle
$$

We then write

$$
\delta_{0}\left\langle\zeta_{c, \vec{k}_{1}} \zeta_{c, \vec{k}_{2}} \zeta_{c, \vec{k}_{3}}\right\rangle=A\left(k_{1}, k_{2}, k_{3}\right) \delta F_{0}\left(k_{1}, k_{2}, k_{3}\right), \quad \delta_{\beta}\left\langle\zeta_{c, \vec{k}_{1}} \zeta_{c, \vec{k}_{2}} \zeta_{c, \vec{k}_{3}}\right\rangle=A\left(k_{1}, k_{2}, k_{3}\right) \delta F_{1}\left(k_{1}, k_{2}, k_{3}\right)
$$

where for the standard scenario

$$
\delta F_{0}^{\text {standard }}=1, \quad \delta F_{1}^{\text {standard }}=0,
$$

so that $A\left(k_{1}, k_{2}, k_{3}\right)$ is indeed the standard three-point function for $\zeta_{c}$.

This representation of the three-point function is useful because in this way one will read from $\delta F_{0}$ and $\delta F_{1}$ the corrections to the standard result, respectively of order $\left|\beta_{k_{i}}\right|^{0}$ and $\left|\beta_{k_{i}}\right|^{1}$, in the modified scenarios, due to the new high energy physics.

\subsubsection{Example 1: standard scenario (review)}

In the standard scenario it is $\eta_{\text {in }}=-\infty$, and the Whightman functions are obtained from (5), (8). We list them in appendix A.2. Inserting them in equation (22), one finds the integral over tims 8

$$
\int_{\eta_{\text {in }}}^{\eta \sim 0} d \eta^{\prime} e^{i\left(k_{1}+k_{2}+k_{3}\right) \eta^{\prime}}\left(k_{1}+k_{2}+k_{3}\right)=-i,
$$

\footnotetext{
${ }^{7}$ In [38, $\zeta_{c}$ is simply written as $\zeta$, see their equation (3.11). The same is done in many other papers, such as [15].

${ }^{8}$ The path of integration in time must be chosen such that the oscillating piece of the integrand becomes exponentially decreasing for $\eta \rightarrow-\infty$. This corresponds to taking the vacuum of the interacting theory [10].
} 
where we have inserted a factor of $k_{t} \equiv k_{1}+k_{2}+k_{3}$ for later comparison with the modified scenarios, see equations (29), (39), and to make the integral dimensionless. The result from (22) is then [10]

$$
A\left(k_{1}, k_{2}, k_{3}\right) \equiv\left\langle\zeta_{c, \vec{k}_{1}} \zeta_{c, \vec{k}_{2}} \zeta_{c, \vec{k}_{3}}\right\rangle_{\mathrm{st}}=4(2 \pi)^{3} \delta^{(3)}\left(\sum_{i} \vec{k}_{i}\right) \frac{H^{6}}{\dot{\phi}^{2} M_{\text {Planck }}^{2}} \frac{k_{1}^{2} k_{2}^{2} k_{3}^{2}}{\prod_{i=1}^{3}\left(2 k_{i}^{3}\right)} \sum_{l} \frac{1}{k_{t} k_{l}^{2}}, \quad k_{t}=\sum_{i=1}^{3} k_{i} .
$$

Adding the disconnected contribution in equation (20) to obtain the bispectrum for $\zeta$, and taking the squeezed limit $k_{1} \ll k_{S}, k_{2,3} \sim k_{S}$, finally [10]

$$
\left\langle\zeta_{\vec{k}_{1}} \zeta_{\vec{k}_{2}} \zeta_{\vec{k}_{3}}\right\rangle_{\mathrm{st}} \underset{k_{1} \ll k_{S}}{=}(2 \pi)^{3} \delta^{(3)}\left(\sum_{i} \vec{k}_{i}\right)\left(1-n_{s}\right) P_{\mathrm{st}}\left(k_{1}\right) P_{\mathrm{st}}\left(k_{S}\right)
$$

at leading order in $\left.\frac{k_{1}}{k_{S}} \ll 1[10] 12\right]$. The result (28) shows Maldacena's consistency condition. The bispectrum in the form (28) is in the so-called local form [10 12.

\subsubsection{Example 1 with modified initial state}

As the computations are simpler in this case, we present it first. We have already discussed before that possible intrinsic non-Gaussianities of the initial condition lead to suppressed and standard-looking results (see section 4 and [15,60,61]). We focus here on the Gaussian part of the initial condition/state, which is responsible for the form of the Whightman functions, and will appear to give rise to much more important and dominant modifications to the squeezed limit of the bispectrum.

It is straightforward to calculate the contribution to the bispectrum from (18) for generic $k_{1}, k_{2}, k_{3}$ in this scenario. The result has been first presented in [38]. Let us quickly review it. We insert the relevant Whightman functions, see appendix $\mathbf{A . 2}$, in (22) and write the result in the form (23), (24) using (27). We also define $k_{t} \equiv k_{1}+k_{2}+k_{3}$, obtaining [38]

$\delta F_{0} \sim 1, \quad \delta F_{1}=\sum_{j=1}^{3} \delta F_{1}^{(j)}=-\sum_{j=1}^{3} \operatorname{Re}\left[i \beta_{k_{j}}^{\mathrm{mis}^{*}} k_{t} \int_{\eta_{c}}^{\eta \sim 0} d \eta^{\prime} e^{i\left(\sum_{h \neq j} k_{h}-k_{j}\right) \eta^{\prime}}\right]=-\sum_{j=1}^{3} \frac{k_{t} \operatorname{Re}\left[\beta_{k_{j}}^{\mathrm{mis}^{*}}\left(1-e^{i\left(\sum_{h \neq j} k_{h}-k_{j}\right) \eta_{c}}\right)\right]}{\sum_{h \neq j} k_{h^{-}} k_{j}}$.

While $\delta F_{0}$ matches the standard result, $\delta F_{1}$ leads to very different outcomes. The presence of a nonzero $\delta F_{1}$ part is a consequence of the negative-frequency component of the Whightman functions. Note the finite-time lower limit of integration at $\eta_{\text {in }}=\eta_{c}$ for the integra 9 .

Starting from this result, we move now to the novel part of the analysis and study the squeezed limit $\left(k_{1} \ll k_{S}, k_{2,3} \sim k_{S}\right)$ for this example of interaction. It appears from (29) that $\delta F_{1}$ is the sum of three contributions. By taking the squeezed limit, we find that the contribution proportional to $\beta_{k_{1}}$ is very small: at leading order

$$
\delta F_{1}^{(1)} \underset{k_{1} \ll k_{S}}{=}-\operatorname{Re}\left[\beta_{k_{1}}^{\mathrm{mis}^{*}}\left(1-e^{i 2 k_{S} \eta_{c}}\right)\right] \ll 1 .
$$

We consider then the contributions proportional to $\beta_{k_{2}}, \beta_{k_{3}}$ in (29), where the perturbations depending on the large momenta in the squeezed limit are in opposition of phase (that is, $k_{j}=k_{2}$ or $k_{3}$ ). These contributions will be much larger than (30). Indeed, expressing $k_{h \neq\{1, j\}}$ in terms of $k_{1}$ and $k_{j}$ as

$$
k_{h \neq\{1, j\}}=\left(k_{1}^{2}+k_{j}^{2}+2 k_{1} k_{j} \cos \theta_{j}\right)^{\frac{1}{2}}
$$

\footnotetext{
${ }^{9}$ The initial conditions for the perturbations $\zeta_{k_{1}}, \zeta_{k_{2}}, \zeta_{k_{3}}$ are fixed respectively at $\eta_{c}^{\left(k_{1}\right)}, \eta_{c}^{\left(k_{2}\right)}, \eta_{c}^{\left(k_{3}\right)}$ when modes are well within the horizon $\left(\left|k_{i} \eta_{c}^{\left(k_{i}\right)}\right| \gg 1, i=1,2,3\right)$. In the BEFT case the initial time is the same for all modes $\left(\eta_{c}\right.$ is independent of $k$, that is, $\eta_{c}^{\left(k_{1}\right)}=\eta_{c}^{\left(k_{2}\right)}=\eta_{c}^{\left(k_{3}\right)}=\eta_{c}$ ). In the NPHS case, instead, the initial times can be different as they depend on the different wavenumbers, therefore the overlap of the perturbations (and so their interaction) is nonzero only after the latest of the initial times. In the squeezed limit, this time is $\eta_{c}^{\left(k_{2}\right)} \sim \eta_{c}^{\left(k_{3}\right)} \sim \eta_{c}^{\left(k_{S}\right)}$. These points are also explained in section 6.3. We will often neglect the ${ }^{\left(k_{S}\right)}$ label to avoid cluttering of formulas.
} 
using momentum conservation, and expanding in $\frac{k_{1}}{k_{j}} \sim \frac{k_{1}}{k_{S}} \ll 1$, one obtains from (29)

$$
\text { for } j=2 \text { or } 3 \quad \sum_{h \neq j} k_{h}-k_{j} \underset{k_{1}}{\underset{\ll k_{2,3}}{\simeq}} k_{1}\left(1+\cos \theta_{j}\right) \equiv k_{1} v_{\theta_{j}} .
$$

so that at leading order

$$
\delta F_{1}^{(j=2,3)} \underset{k_{1} \ll k_{S}}{=}-\frac{k_{1}+k_{2}+k_{3}}{k_{1} v_{\theta_{j}}} \operatorname{Re}\left[\beta_{k_{j}}^{\mathrm{mis}^{*}}\left(1-e^{i k_{1} \eta_{c} v_{\theta_{j}}}\right)\right] .
$$

It can be easily checked (for example by plotting) that the one in (32) is an almost perfect approximation for all values of $\theta_{j}$ already for $\frac{k_{1}}{k_{j}} \sim \frac{k_{1}}{k_{S}} \sim 0.1$.

The form of the correction $\delta F_{1}^{(j=2,3)}$, then, depends on the interplay between $k_{1}, \eta_{c}$ and $v_{\theta_{j}}$. Recalling that we consider the realistic limit, where $k_{1}$ is small but not zero, there are two asymptotic possibilities where the result can be evaluated most explicitly. From (33), at leading order

$$
\begin{array}{lll}
1)\left|k_{1} \eta_{c} v_{\theta_{j}}\right| \gg 1 & \Rightarrow & \delta F_{1}^{(j=2,3)} \underset{k_{1} \ll k_{S}}{=}-2 \frac{k_{S}}{k_{1}} v_{\theta_{j}}^{-1} \operatorname{Re}\left[\beta_{k_{S}}^{\mathrm{mis}^{*}}\right] \\
2)\left|k_{1} \eta_{c} v_{\theta_{j}}\right| \ll 1 & \Rightarrow \quad \delta F_{1}^{(j=2,3)} \underset{k_{1} \ll k_{S}}{=}-2 k_{S} \eta_{c} \operatorname{Im}\left[\beta_{k_{S}}^{\mathrm{mis}^{*}}\right],
\end{array}
$$

where in the case 1) we have eventually taken into account that in standard observables such as the halo bias, see section 7 , the final result involves an integration/average over quantities such as $\theta_{j}, k_{S}$ and so the terms with large oscillations average to zero.

We see that (34), (35) dominate over (30) because of the large factors $\frac{k_{S}}{k_{1}}$ and $k_{S} \eta_{c}$. This result is due to the interference leading to phase cancellation in the integrand of (29), and the cumulative effect due to the time integration. Comparing to (26), we see that the latter is instead ineffective in the standard scenario, where the result is dominated by the upper limit of integration (late time). It is also clear why intrinsic non-Gaussianities of the initial condition would have a different form and be subdominant: in that case there is no time-integration, as intrinsic non-Gaussianities are nonzero at the initial time only.

Thus, the leading correction to the squeezed limit of the three-point function for nonzero $\beta_{k}$ in the case of the "minimal cubic coupling" (18), neglecting subleading corrections in $\frac{k_{1}}{k_{S}} \ll 1$, reads

$$
\delta_{\beta}\left\langle\zeta_{c, \vec{k}_{1}} \zeta_{c, \vec{k}_{2}} \zeta_{c, \vec{k}_{3}}\right\rangle_{\text {min cub }}^{\mathrm{mis}} \equiv A\left(k_{1}, k_{2}, k_{3}\right) \delta F_{1}\left(k_{1}, k_{2}, k_{3}\right)_{k_{1} \ll k_{S}}^{=}(2 \pi)^{3} \delta^{(3)}\left(\sum_{i} \vec{k}_{i}\right) \mathcal{B}_{\text {min cub }}^{\text {mis }} P_{\text {st }}\left(k_{1}\right) P_{\text {st }}\left(k_{S}\right),
$$

with

$$
\mathcal{B}_{\text {min cub }}^{\text {mis }}=\sum_{j=2}^{3} \mathcal{B}_{(j)}^{\text {mis, min cub }}, \quad \mathcal{B}_{(j)}^{\text {mis, min cub }}= \begin{cases}-4 \epsilon \frac{k_{S}}{k_{1}} v_{\theta_{j}}^{-1} \operatorname{Re}\left[\beta_{k_{S}}^{\text {mis* }^{*}}\right] & \text { if }\left|k_{1} \eta_{c} v_{\theta_{j}}\right| \gg 1 \\ -4 \epsilon k_{S} \eta_{c} \operatorname{Im}\left[\beta_{k_{S}}^{\text {mis }^{*}}\right] & \text { if }\left|k_{1} \eta_{c} v_{\theta_{j}}\right| \ll 1\end{cases}
$$

For observational purposes, for example concerning the halo bias, one is interested in the magnitude of these corrections and in their dependence on the probed large scale $k_{1}^{-1}$. The $k_{1}$-dependence of the leading contributions (36), (37) is fully determined, since $\beta_{k_{S}}^{\text {mis }}$ depends on $k_{S}$ and not $k_{1}$. The magnitude will have to be estimated and bound using the phenomenological constraints reviewed in section 3.4

In section [6 we will discuss in details the features of this result (and of the more general results we will obtain later on), and see if the different models of modified initial condition favour case 1) or 2) in (34), (35). For the moment, the main evident differences compared to the standard scenario are as follows. For $\left|k_{1} \eta_{c} v_{\theta_{j}}\right| \ll 1$ the result has the same dependence on $k_{1}$ and $1-n_{s} \sim \epsilon$ as the standard one, but with a new amplitude factor $\left|k_{S} \eta_{c}\right| \gg 1$. Instead, for $\left|k_{1} \eta_{c} v_{\theta_{j}}\right| \gg 1$, the $k_{1}$-dependence of this and the standard result are different. 


\subsubsection{Example 1 with modified dispersion relations}

The contribution to the bispectrum in this case was first computed in [20] for generic $k_{1}, k_{2}, k_{3}$. Before discussing the squeezed limit, we briefly review the computation, addressing the reader to [20] for more details.

The three-point function (22) in this scenario is computed using the relevant Whightman functions listed in appendix A.2. The latter ones are obtained using the field solution (11) in the general definition (5). Let us also recall that the error induced by the approximations in solving the equation for the mode functions is calculable and can be made small at will by going to higher order in the approximations. The results for the bispectrum are robust against that (small) error, see [20].

The time integral in the bispectrum (22) can then be divided into the different intervals of validity of the piecewise solutions (111). In [20, it was shown that the leading contribution to the bispectrum occurs when the Whightman functions have support in the intervals IV and III, see figure 1. Other contributions are indeed suppressed by $\Delta$, defined in equation (12), or by a rapidly decaying integrand 10 , see [20]. We concentrate, therefore, only on the leading contributions from intervals IV and III.

It is convenient to write the result in terms of the variables

$$
y \equiv-\frac{p_{\max }(\eta)}{\Lambda}=\frac{H}{\Lambda} k_{\max } \eta, \quad x_{i} \equiv \frac{k_{i}}{k_{\max }} .
$$

In particular, when taking the squeezed limit it will be $k_{\max } \approx k_{2,3} \approx k_{S}$ and so in these variables the limit reads $x_{1} \ll 1, x_{2,3} \simeq x_{S} \simeq 1$. Since $y$ is a rescaled time coordinate, sometimes we will call it simply "time" in this section.

By inserting in equation (22) the relevant Whightman functions, listed in appendix A.2, and writing the result in the form (23), (24) using (27), the corrections $\delta F_{m=\{0,1\}}$ read [20]

$$
\delta F_{m=\{0,1\}}\left(x_{1}, x_{2}, x_{3}, y\right)=\sum_{j=1}^{3} \operatorname{Re}\left[\left(\beta_{k_{j}}^{\mathrm{mdr}^{*}}\right)^{m} \frac{\Lambda}{H} \int_{y_{\mathrm{II}}}^{y} d y^{\prime} x_{t} g\left(\left\{x_{h \neq j}\right\}, x_{j}, y^{\prime}, m\right) e^{i \frac{\Lambda}{H} S_{0}\left(\left\{x_{h \neq j}\right\}, x_{j}, y^{\prime}, m\right)}\right],
$$

where $x_{t} \equiv \sum_{i=1}^{3} x_{i}$, and $g\left(\left\{x_{h \neq j}\right\}, x_{j}, y^{\prime}, m\right)$ is reported in equation (111) of appendix A.3. $S_{0}$ is given by 11

$$
S_{0}\left(\left\{x_{h \neq j}\right\}, x_{j}, y^{\prime}, m\right)=\int^{y^{\prime}} d y^{\prime \prime}\left(\sum_{h \neq j} \omega\left(x_{h}, y^{\prime \prime}\right)+(-1)^{m} \omega\left(x_{j}, y^{\prime \prime}\right)\right), \quad h, j \in\{1,2,3\},
$$

The limits of integration for the variable $y^{\prime}$ in (39) have been discussed in [20], which we refer the reader to for the details, while reporting here the results. The upper limit is $y \approx 0$ because the bispectrum is evaluated at late time $\eta \approx 0$, while the lower limit $y_{\text {II }}$ is determined by the smallest among $\eta_{\mathrm{II}}^{\left(k_{i=1,2,3}\right)}$, which bound region III for the three momenta $k_{i=1,2,3}$, see equation (11). As shown in [20], it is $y_{\mathrm{II}}=\frac{H}{\Lambda} k_{\max } \eta_{\mathrm{II}}^{\left(k_{\max }\right)} \approx-1$ 12. Observe also that, in the squeezed limit, $\left|x_{1} y\right| \leq\left|x_{1} y_{\mathrm{II}}\right| \ll 1$ $\forall|y| \leq\left|y_{\text {II }}\right|$, so effectively $F\left(\frac{H}{\Lambda} k_{1} \eta\right)=F\left(x_{1} y\right) \simeq 1$ and $\omega\left(x_{1} y\right) \simeq x_{1}$.

\footnotetext{
${ }^{10}$ Recall that the prescription in footnote 8 must be followed also here.

${ }^{11}$ Observe that $\omega(x, y) \equiv x F(x y)$ is dimensionless.

${ }^{12}$ This is indeed straightforward: the corrections to the linear dispersion relation at the time $\eta_{\text {II }}^{(k)}$ that separates region III and II must be quite important in order to drive the frequency close to the turning point (see figure 1). As these corrections grow as powers of $-\frac{p(\eta)}{\Lambda}$, see (10), it must then be $\left|p\left(\eta_{\mathrm{II}}^{(k)}\right) \Lambda^{-1}\right| \sim 1$. Furthermore, since $p\left(\eta_{\mathrm{II}}^{(k)}\right)=-k \eta_{\mathrm{II}}^{(k)} H$, the relevant boundary time $\eta_{\mathrm{II}}^{(k)}$ (the smallest, as we recalled) is the one for the largest $k$, which is indeed $k_{\max }$, and thus, from the definition of $y$, it follows that $\left|y_{\text {II }}\right|=\left|p_{\max }\left(\eta_{\text {II }}^{\left(k_{\max }\right)}\right) \Lambda^{-1}\right| \sim 1$.
} 
We move now to the novel part of the analysis and study the squeezed limit. The integral in (39) can be conveniently written as the sum of two parts, respectively when the Whightman functions depending on $k_{2,3}$ have support in their regions IV or III.

We begin studying $\delta F_{m=0}$. In region IV $\zeta_{k_{2}}$ and $\zeta_{k_{3}}$ are superhorizon, and the contribution to the bispectrum is straightforward to calculate using the Whightman functions listed in appendix A.2. In region III, instead, the integral in equation (39), which is a typical Fourier integral, is dominated by the contributions of points where the strong suppression $\left(\frac{\Lambda}{H} \gg 1\right)$ from the oscillating phase of the integrand is reduced. This happens for stationary points of $S_{0}$ and boundary points [20,69].

But in the case of $\delta F_{m=0}$ there are no stationary points. Indeed, from (40) we see that for $m=0$ the first derivative of $S_{0}\left(x_{1,2,3}, y^{\prime}, m=0\right)$ is the sum of the positive-defined frequencies and thus is always positive and never zero [20]. Then, in this case the Fourier integrals can be approximated for $\frac{\Lambda}{H} \gg 1$ by integrating by parts (see [69] and section 4.1.2 in [20]).

Putting together all the contributions and using the information in appendix A.3, we find that in the squeezed limit, neglecting subleading corrections,

$$
\delta F_{0} \underset{\substack{k_{1} \ll k_{S} \\\left(x_{1} \ll 1\right)}}{=} 1-\left.\frac{1}{\omega(1,-1)} \operatorname{Im}\left[g\left(x_{1}, x_{2}, x_{3},-1,0\right) e^{i \frac{\Lambda}{H} S_{0}\left(x_{1}, x_{2}, x_{3},-1,0\right)}\right]\right|_{\substack{x_{1} \simeq 0 \\ x_{2,3} \simeq x_{S} \simeq 1}} \simeq 1,
$$

where in the last passage, as before, we have taken into account the averaging to zero of the rapid oscillations in observables such as the halo bias. As we see, comparing to the first one of (25), this contribution does not lead to very significant corrections to the standard local form of the bispectrum.

We expect a more interesting behaviour for what concerns $\delta F_{m=1}$. We start by discussing the contribution from region IV of $\zeta_{k_{2,3}}$ (the region where $\zeta_{k_{2,3}}$ are superhorizon). In the squeezed limit, that contribution is very much subdominant. This is because those modes become superhorizon only at $\eta_{S} \simeq-k_{2,3}^{-1} \simeq-k_{\max }^{-1}$, that is, using (38), for $y_{S}=-\frac{H}{\Lambda} \sim 0$. This time is then very close to the late time $y \sim 0$ that the bispectrum is evaluated at. Computing then the contribution of region IV from (39), we find that it is of order $\beta$, hence very small.

We turn now to the contribution from region III of $\zeta_{k_{2,3}}$. From (39) and (40) for $m=1$, we see that in the squeezed limit $x_{1} \ll 1$ the dominant contributions are those where the mode function in the negative-energy branch is the one depending on $k_{2}$ or $k_{3}$. The reason is that in those cases the suppression from the oscillations of the integrand is very reduced, because the perturbations depending on the large momenta are in opposition of phase and the overall phase of the integrand becomes very small. Indeed,

for $j=2$ or 3 :

$$
\begin{aligned}
S_{0}\left(\left\{x_{h \neq j}\right\}, x_{j}, y^{\prime}, 1\right) & =\int^{y^{\prime}} d y^{\prime \prime}\left(\sum_{h \neq j} \omega\left(x_{h}, y^{\prime \prime}\right)-\omega\left(x_{j}, y^{\prime \prime}\right)\right) \underset{x_{1} \ll 1}{\simeq} x_{1} \int^{y^{\prime}} d y^{\prime \prime}\left(1+\left.\partial_{x} \omega\left(x, y^{\prime \prime}\right)\right|_{x_{S}} \cos \theta_{j}\right)+\mathcal{O}\left(x_{1}^{2}\right) \\
& =x_{1} y^{\prime}\left(1+F\left(y^{\prime}\right)\right) \cos \left(\theta_{j}\right)+\mathcal{O}\left(x_{1}^{2}\right) \equiv x_{1} \tilde{v}_{\theta_{j}}\left(y^{\prime}\right)
\end{aligned}
$$

using equations (10), (31) and $x_{s} \simeq 1$ ( $\mathcal{O}$ is Landau's big-O symbol).

Inserting (42) back in equation (39), the integral has then two asymptotic regimes [69], depending on the magnitude of the integrand's phase factor $\frac{\Lambda}{H} x_{1}$. Using the information listed in appendix A.3. 
table 1, we find at leading order

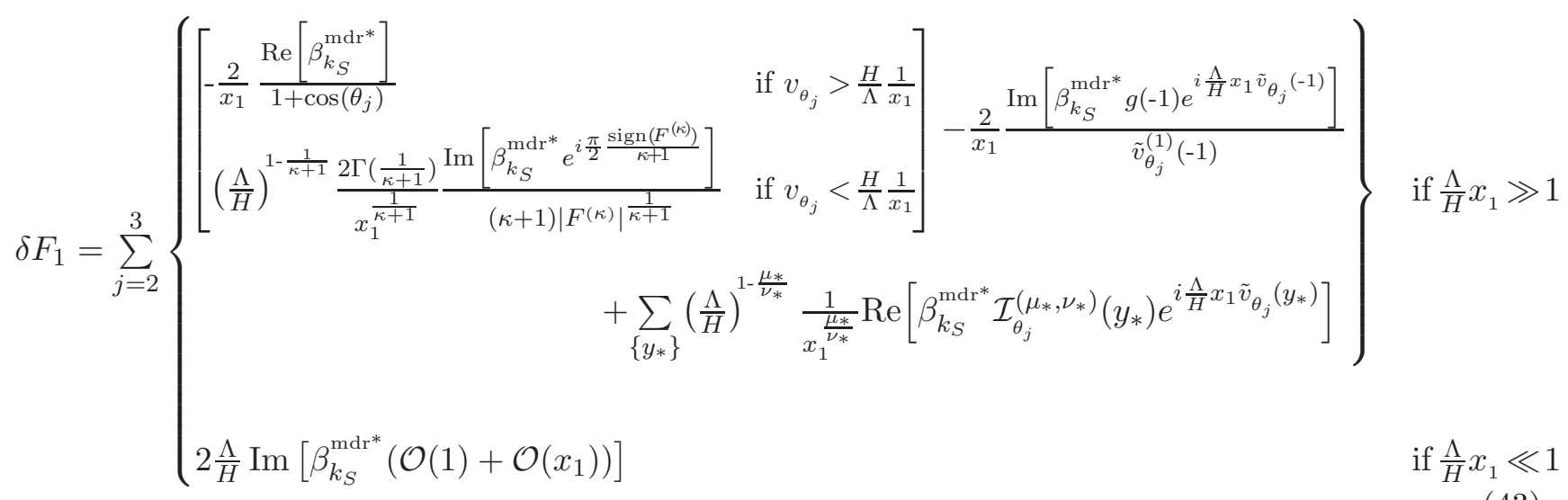

where we have defined

$$
\mathcal{I}_{\theta_{j}}^{\left(\mu_{*}, \nu_{*}\right)}\left(y_{*}\right)=\frac{\Gamma\left(\frac{\mu_{*}}{\nu_{*}}\right)}{\nu_{*}\left(\left|\tilde{v}_{\theta_{j}}^{\left(\nu_{*}\right)}\left(y_{*}\right)\right|\right)^{\frac{\mu_{*}}{\nu_{*}}}} g^{\left(\mu_{*-1}\right)}\left(y_{*}\right)\left(\sum_{l=0}^{1}(-1)^{l\left(\mu_{*}-1\right)} e^{( \pm 1)^{l} i \frac{\pi}{2} \frac{\mu_{*}}{\nu_{*}} \operatorname{sign}\left(\tilde{v}_{\theta_{j}}^{\left(\nu_{*}\right)}\left(y_{*}\right)\right)}\right),
$$

and the $\mathcal{O}(1)$ coefficient in the last line of (43) is reported in detail in equation (114), appendix A.3.

Equation (43) is the complete leading-asymptotic result of the integral. It is a complicated formula, which we write for illustrative purposes, but the final result will be simpler and neat. We have introduced some new notation, which we explain here below as well as in appendix A.3.

First of all, let us discuss the contribution from the possible presence of stationary points, which appear in the second line of (43), case $\frac{\Lambda}{H} x_{1} \gg 1$. We have indicated those points by $\left\{y_{*}\right\}$, and their orders of stationariness for the function (42) are given by the numbers $\left\{\nu_{*}\right\}$. In general terms, they are also zeros of orders $\left\{\mu_{*}-1\right\}$ for the function $g$ in (39) 13 .

The presence of stationary points is obviously model-dependent. However, their contributions will be suppressed in the final observables such as the halo bias, because of the averaging to zero of the large oscillations due to their phase, visible in (43). Thus, at the end we can neglect them, and they do not spoil the generality of the final results we will obtain.

We are left then with the other contributions in (43), which are always present 69. Note that in the case of the contribution from the (nearly) folded configurations (the one for $v_{\theta_{j}} \equiv 1+\cos \left(\theta_{j}\right)<\frac{H}{\Lambda} \frac{1}{x_{1}}$ in (43)), one finds that the scaling is determined by the lowest-order correction to the dispersion relation (see appendix A.3, table 10. That is, $F^{(\kappa)} \sim \mathcal{O}(1)$ and $\kappa$ in (43) enter the expansion (10)

$$
\omega_{\text {phys }}(p) \sim p\left(1+\left(\frac{p}{\Lambda}\right)^{\kappa} F^{(\kappa)}+\cdots\right),
$$

thus they capture the leading correction to the dispersion relation of the specific models.

Finally, from (23), using (24), (27) (41), (43), the leading correction to the standard consistency relation due to nonzero $\beta_{k}$ in the case of the "minimal cubic coupling" (18) reads

$$
\delta_{\beta}\left\langle\zeta_{c, \vec{k}_{1}} \zeta_{c, \vec{k}_{2}} \zeta_{c, \vec{k}_{3}}\right\rangle_{\min \text { cub }}^{\mathrm{mdr}} \bar{k}_{1} \overline{\bar{k}}_{S}(2 \pi)^{3} \delta^{(3)}\left(\sum_{i} \vec{k}_{i}\right) \mathcal{B}_{\min \mathrm{mub}}^{\mathrm{mdr}} P_{\mathrm{st}}\left(k_{1}\right) P_{\mathrm{st}}\left(k_{S}\right),
$$

\footnotetext{
${ }^{13}$ The coefficients of the leading behaviour of the functions $\tilde{v}_{\theta_{j}}(y), g(\{x\}, y)$ in the squeezed limit in proximity of a stationary point $y_{*}$ have been written as $\tilde{v}_{\theta_{j}}^{\left(\nu_{*}\right)}\left(y_{*}\right), g^{\left(\mu_{*}-1\right)}\left(y_{*}\right)$, see table 1 in appendix A.3 and (44). In particular, if $\nu_{*}$ is an integer, then $\tilde{v}_{\theta_{j}}^{(n)}=n !^{-1} \partial_{y}^{n} \tilde{v}_{\theta_{j}}$ (similarly for $\mu_{*}$ and $g^{\left(\mu_{*-1}\right)}$ ). The sign \pm in the phases in (44) depends on $\tilde{v}_{\theta_{j}}(y)-\tilde{v}_{\theta_{j}}\left(y_{*}\right)$ being even or odd under $\left(y-y_{*}\right) \rightarrow-\left(y-y_{*}\right)$, for $y \sim y_{*}$.
} 


$$
\mathcal{B}_{\text {min cub }}^{\text {mdr }}=\sum_{j=2}^{3}\left\{\begin{array}{l}
{\left[\begin{array}{l}
-4 \epsilon \frac{k_{S}}{k_{1}} \frac{1}{1+\cos \left(\theta_{j}\right)} \operatorname{Re}\left[\beta_{k_{S}}^{\mathrm{mdr} *}\right] \\
4 \epsilon\left(\frac{\Lambda}{H}\right)^{1-\frac{1}{\kappa+1}}\left(\frac{k_{S}}{k_{1}}\right)^{\frac{1}{\kappa+1}} \Gamma\left(\frac{1}{\kappa+1}\right) \frac{\operatorname{Im}\left[\beta_{k_{S}}^{\mathrm{mdr}_{S}^{*}} e^{i \frac{\pi}{2} \frac{\operatorname{sign}\left(F^{(\kappa)}\right)}{\kappa+1}}\right]}{(\kappa+1)\left|F^{(\kappa)}\right|^{\frac{1}{\kappa+1}}} \\
4 \epsilon \frac{\Lambda}{H} \operatorname{Im}\left[\beta_{k_{S}}^{\mathrm{mdr}^{*}} \mathcal{O}(1)\right]
\end{array}\right.}
\end{array}\right.
$$

$$
\begin{aligned}
\text { if } \left.1+\cos \left(\theta_{j}\right)>\frac{H}{\Lambda} \frac{1}{x_{1}}\right] & \\
\text { if } \left.1+\cos \left(\theta_{j}\right)<\frac{H}{\Lambda} \frac{1}{x_{1}}\right] & \\
& \text { if } \frac{\Lambda}{H} x_{1} \ll 1
\end{aligned}
$$

where we have accounted for the averaging to zero of the rapid oscillations in (43) in observables such as the halo bias. Recall that the $\mathcal{O}(1)$ coefficient in the last line is specified in (114) appendix A.3.

Deferring comments to section 6, for the moment we just note that, once again, at leading order the dependence on $k_{1}$ of the result is fully specified, and there appear enhancement factors such as $\frac{\Lambda}{H}$ and $\frac{k_{S}}{k_{1}}$ thanks to interference and accumulation in time. Finally, the only relevant sensitivity to the specific models, a part from $\beta_{k_{S}}^{\text {mdr* }}$, is due to $F^{(\kappa)} \sim \mathcal{O}(1)$ and $\kappa$, which capture the leading correction to the dispersion relation of the specific models, and thus are interesting for detection.

The final result (46), (47) is very similar to the one obtained in the modified initial state case. This points at the fact that, indeed, at leading order the squeezed limit is dominated by the generic features common to both scenarios (such as particle content/creation, interference effects). The only difference is that in the modified initial state case, the folded configurations (given by $\sum_{h \neq j} k_{h}-k_{j}=$ 0 , that is, $v_{\theta_{j}}=0$ ) yield a contribution corresponding to $\kappa \rightarrow \infty$ in (47), since the phase of the integrand and all its derivatives are identically zero, see the second line in (37). In the case of modified dispersion relations, instead, the true analogous of the folded configuration (which would be $\sum_{h \neq j} \omega\left(x_{h}, y^{\prime}\right)-\omega\left(x_{j}, y^{\prime}\right)=0$ for some $x$ 's but for all $y^{\prime}$ 's) does not occur 14 and $\kappa$ is finite.

\subsection{Example 2: Quartic derivative interaction}

In this section, we present our second detailed example before passing to the more general discussion in section 5.3. We consider the higher-derivative cubic interaction coming from the correction $\mathcal{L}_{\text {HDI }}=$ $\sqrt{-\operatorname{det} G} \frac{g}{8 \Lambda^{4}}\left((\nabla \Phi)^{2}\right)^{2}$ to the effective action of the inflaton $\Phi$, where $G$ is the metric, $g$ the coupling.

Expanding in perturbations and converting in terms of $\zeta$ as explained in details in [20,38], from $\mathcal{L}_{\text {HDI }}$ one obtains the cubic term

$$
H_{(I)}=-\int d^{3} x a \frac{g \dot{\phi}^{4}}{2 H^{3} \Lambda^{4}} \zeta^{\prime}\left(\zeta^{\prime 2}-\left(\partial_{i} \zeta\right)^{2}\right)
$$

Because of its origins from the term $\mathcal{L}_{\text {HDI }}$, which contains quartic power of derivatives, we call this cubic interaction "quartic derivative interaction". It is allowed to treat these interactions perturbatively also when the physical momenta $p=a^{-1} k$ are such that $\frac{p}{\Lambda}$ is not small any more, because, compared to the operators changing the dispersion relation, they are further suppressed by additional powers of the perturbation fields.

In the standard scenario, the contributions to the bispectrum from higher derivative interactions are suppressed in the squeezed limit by powers of $\frac{k_{1}}{k_{S}}$ [12]. It is interesting, then, to study if one finds the same suppressed contributions and the same overall scale dependence in the scenarios of modified initial state and modified dispersion relations. In the standard single-field slow-roll scenario, reference [68] has discussed that the one in (48) is the lowest-dimensional operator in the class of operators that is the most important for non-Gaussianities. Nonetheless, in the standard scenario its contribution is very suppressed in the squeezed limit, as we have said.

\footnotetext{
${ }^{14}$ It can be easily verified at least if $\omega$ is given by elementary functions and/or power series.
} 
Using equations (44), the bispectrum for the coupling (48) reads

$$
\begin{aligned}
\left\langle\zeta_{\vec{k}_{1}}(\eta) \zeta_{\vec{k}_{2}}(\eta) \zeta_{\vec{k}_{3}}(\eta)\right\rangle=2 \operatorname{Re} & {\left[-i(2 \pi)^{3} \delta^{(3)}\left(\sum_{i} \vec{k}_{i}\right) \frac{g \dot{\phi}^{4}}{2 H^{3} \Lambda^{4}} \int_{\eta_{i n}}^{\eta} d \eta^{\prime} a\left(\partial_{\eta} G_{k_{1}}\left(\eta, \eta^{\prime}\right) \partial_{\eta} G_{k_{2}}\left(\eta, \eta^{\prime}\right) \partial_{\eta} G_{k_{3}}\left(\eta, \eta^{\prime}\right)+\right.\right.} \\
+ & \left.\left.\vec{k}_{1} \cdot \vec{k}_{2} G_{k_{1}}\left(\eta, \eta^{\prime}\right) G_{k_{2}}\left(\eta, \eta^{\prime}\right) \partial_{\eta} G_{k_{3}}\left(\eta, \eta^{\prime}\right)\right)+ \text { permutations }\right]
\end{aligned}
$$

where, again, $\eta \sim 0$ is a very late time when all modes $k_{i}$ are outside the horizon.

\subsubsection{Example 2 with modified initial state}

In this scenario the leading contribution to the bispectrum from (49) for generic external momenta has been computed in [15, 38. Writing (49) in the same form as (23), (24), using (27), the main modifications compared to the standard result come from $\delta F_{1}$, which reads

$$
\delta F_{1}\left(k_{1}, k_{2}, k_{3}, \eta \simeq 0\right)=\operatorname{Re}\left[i C \frac{k_{S}^{3}}{k_{1}^{2} k_{2}^{2} k_{3}^{2}} \sum_{j=1}^{3} \beta_{k_{j}}^{\text {mis* }^{*}} \int_{\eta_{c}}^{0} d \eta^{\prime} e^{i\left(\sum_{h \neq j} k_{h}-k_{j}\right) \eta^{\prime}} P_{j}\left(k_{1}, k_{2}, k_{3}, \eta^{\prime}\right)+\text { c.c. }\right]
$$

with

$$
\begin{gathered}
C \equiv \frac{x_{t} \prod_{l=1}^{3} x_{l}^{2}}{\sum_{r>v} x_{r}^{2} x_{v}^{2}} \frac{g H^{2} M_{\text {Planck }}^{2}}{4 \Lambda^{4}}, \\
P_{j}\left(k_{1}, k_{2}, k_{3}, \eta^{\prime}\right)=-k_{t} \prod_{i=1}^{3}\left(\sum_{h \neq i} k_{h}-k_{i}\right)-i \eta^{\prime}\left(\left(k_{j+1}^{2}-k_{j}^{2}-k_{j+2}^{2}\right) k_{j+1}^{2}\left(k_{j+2}-k_{j}\right)+\left(k_{j+2}^{2}-k_{j}^{2}-k_{j+1}^{2}\right)\right. \\
\left.k_{j+2}^{2}\left(k_{j+1}-k_{j}\right)+\left(k_{j}^{2}-k_{j+1}^{2}-k_{j+2}^{2}\right) k_{j}^{2}\left(k_{j+1}+k_{j+2}\right)\right)+\left(-\eta^{\prime}\right)^{2}\left(\sum_{h \neq j} k_{h}-k_{j}\right)\left(\prod_{i=1}^{3} k_{i}\right)\left(k_{t}^{2}-4 k_{j+1} k_{j+2}\right),
\end{gathered}
$$

where $j$ is defined modulo 3 . The factor $C$ has been written in terms of the variables $x_{i}$ defined in equation (38).

Now we study the squeezed limit. As in section [5.1.2, we see from (50) that in that limit the dominating contributions to the integral occur for $j=2,3$ in equation (50), when the perturbations depending on the large wavenumbers are in opposition of phase, so that the overall phase of the integrand is small and the suppression due to the oscillations is reduced. It is then straightforward to see that the largest contributions come from the terms of order $\eta$ and $\eta^{2}$ in $P_{j}$. Indeed, their integration yields

$$
\begin{aligned}
& j=2,3 \quad \int_{\eta_{c}}^{0} d \eta^{\prime} e^{i\left(\sum_{h \neq j} k_{h}-k_{j}\right) \eta^{\prime}} \eta^{\prime}=\frac{1+e^{i k_{1} \eta_{c} v_{\theta_{j}}}\left(-1+i k_{1} \eta_{c} v_{\theta_{j}}\right)}{k_{1}^{2} v_{\theta_{j}}^{2}} \equiv \frac{I_{1}^{(j)}}{k_{1}^{2} v_{\theta_{j}}^{2}}, \\
& j=2,3 \quad \int_{\eta_{c}}^{0} d \eta^{\prime} e^{i\left(\sum_{h \neq j} k_{h}-k_{j}\right) \eta^{\prime}} \eta^{\prime 2}=i \frac{2+e^{i k_{1} \eta_{c} v_{\theta_{j}}}\left(-2+2 i k_{1} \eta_{c} v_{\theta_{j}}+k_{1}^{2} \eta_{c}^{2} v_{\theta_{j}}^{2}\right)}{k_{1}^{3} v_{\theta_{j}}^{3}} \equiv \frac{I_{2}^{(j)}}{k_{1}^{3} v_{\theta_{j}}^{3}},
\end{aligned}
$$

so that at leading order

$$
\delta F_{1} \underset{k_{1} \ll k_{S}}{=} \sum_{j=2}^{3} \frac{2 g H^{2} M_{\text {Planck }}^{2}}{\Lambda^{4}} \frac{k_{S}}{k_{1}} v_{\theta_{j}}^{-2}\left(-\operatorname{Im}\left[\beta_{k_{j}}^{\text {mis* }^{*}} I_{2}^{(j)}\right]-\cos \left(\theta_{j}\right) \operatorname{Re}\left[\beta_{k_{j}}^{\text {mis* }^{*}} I_{1}^{(j)}\right]\right)
$$


Looking at (53), (54), we find again two asymptotic behaviours for the correction to the squeezed limit of the three-point function depending on whether $\left|k_{1} \eta_{c} v_{\theta_{j}}\right| \gg 1$ or $\left|k_{1} \eta_{c} v_{\theta_{j}}\right| \ll 1$. Therefore, at leading order we obtain, for the "quartic derivative" coupling (48),

$$
\delta_{\beta}\left\langle\zeta_{\vec{k}_{1}} \zeta_{\vec{k}_{2}} \zeta_{\vec{k}_{3}}\right\rangle_{\text {quart der }}^{\text {mis }} \underset{k_{1} \ll k_{S}}{=}(2 \pi)^{3} \delta^{(3)}\left(\sum_{i} \vec{k}_{i}\right) \mathcal{B}_{\text {quart der }}^{\text {mis }} P_{\text {st }}\left(k_{1}\right) P_{\text {st }}\left(k_{S}\right),
$$

with

$$
\mathcal{B}_{\text {quart der }}^{\text {mis }}=\sum_{j=2}^{3} \begin{cases}-4 \epsilon \frac{g H^{2} M_{\text {Planck }}^{2}}{\Lambda^{4}} \frac{k_{S}}{k_{1}} v_{\theta_{j}}^{-2}\left(2+\cos \left(\theta_{j}\right)\right) \operatorname{Re}\left[\beta_{k_{S}}^{\text {mis* }}\right] & \text { if }\left|k_{1} \eta_{c} v_{\theta_{j}}\right| \gg 1 \\ 2 \epsilon \frac{g H^{2} M_{\text {Planck }}^{2}}{\Lambda^{4}} \frac{k_{1}}{k_{S}}\left(k_{S} \eta_{c}\right)^{2}\left(\frac{2}{3} \frac{k_{1}}{k_{S}}\left(k_{S} \eta_{c}\right) v_{\theta_{j}} \operatorname{Im}\left[\beta_{k_{S}}^{\text {mis }}\right]+\cos \left(\theta_{j}\right) \operatorname{Re}\left[\beta_{k_{S}}^{\text {mis }}\right]\right) & \text { if }\left|k_{1} \eta_{c} v_{\theta_{j}}\right| \ll 1 .\end{cases}
$$

In the first of (57), we have again taken into account the averaging of large oscillations to zero in observables such as the halo bias. Recall that $\left|k_{S} \eta_{c}\right| \gg 1$ (in the NPHS scenario, in particular, $\left.\left|k_{S} \eta_{c}\right|=\frac{\Lambda}{H}\right)$. We comment on the actual magnitude and scale dependence of these corrections to the bispectrum in section 6 ,

\subsubsection{Example 2 with modified dispersion relations}

In this scenario the three-point scalar correlator from (49) for generic $k_{1}, k_{2}, k_{3}$ was computed in 20]. We briefly review that computation. The dominant contribution occurs when the Whightman functions have support in regions IV/III, see (11), as shown in [20] and reviewed in section 5.1.3. Inserting in equation (49) the relevant Whightman functions listed in appendix A.2, and writing the result as in (23), (24) using (27), with the variables defined in (38), one obtains that the leading modification compared to the standard result is [20]

$$
\delta F_{1}=-\sum_{j} \operatorname{Re}\left[C\left(\frac{\Lambda}{H}\right)^{3} \beta_{k_{j}}^{\mathrm{mdr}^{*}} \int_{y_{\mathrm{I}}}^{y} d y^{\prime} y^{\prime 2} e^{i \frac{\Lambda}{H} S_{0}\left(\left\{x_{h}\right\}, x_{j}, y^{\prime}, 1\right)} q\left(\left\{x_{h}\right\}, x_{j}, y^{\prime}\right)\right] \quad h \neq j,
$$

where the limits of integration are those presented in section 5.1.3. The function $q$ is reported in equation (115) of appendix A.3, while $S_{0}$ and $C$ are defined respectively in equation (40) and (51).

Now we study the squeezed limit. As we see, the correction (58) is again given by a Fourier integral. Once again, the suppression from the oscillations of the integrand is reduced when the perturbations depending on $k_{2}, k_{3}$ are in opposition of phase. Then, $S_{0}\left(\left\{x_{1,2(3)}\right\}, x_{3(2)}, y^{\prime}, 1\right)$ behaves as in equation (42). The integral can be solved by asymptotic techniques, and we obtain at leading order

$$
\delta_{\beta}\left\langle\zeta_{\vec{k}_{1}} \zeta_{\vec{k}_{2}} \zeta_{\vec{k}_{3}}\right\rangle_{\text {quart der }}^{\text {mdr }} \sum_{1} \bar{\aleph}_{S}(2 \pi)^{3} \delta^{(3)}\left(\sum_{i} \vec{k}_{i}\right) \mathcal{B}_{\text {quart der }}^{\text {mdr }} P_{\text {st }}\left(k_{1}\right) P_{\text {st }}\left(k_{S}\right)
$$

with (recalling appendix A.3 and table 1)

$$
\begin{aligned}
\mathcal{B}_{\text {quart der }}^{\text {mdr }}= & -4 \epsilon \frac{g H^{2} M_{\text {Planck }}^{2}}{\Lambda^{4}} \\
& \times \sum_{j=2}^{3}\left\{\begin{array}{lr}
{\left[\frac{k_{S}}{k_{1}} \frac{\Gamma(3)+\cos \left(\theta_{j}\right)}{\left(1+\cos \left(\theta_{j}\right)\right)^{2}} \operatorname{Re}\left[\beta_{k_{S}}^{\mathrm{mdr} *}\right]\right.} & \text { if } v_{\theta_{j}}>\frac{H}{\Lambda} \frac{1}{x_{1}} \\
\frac{\Lambda}{H}\left(\frac{\Lambda}{H} \frac{k_{1}}{k_{S}}\right)^{1-\frac{2}{\kappa+1}} \frac{\Gamma\left(\frac{\kappa+3}{\kappa+1}\right)}{(\kappa+1)} \frac{2 \operatorname{Im}\left[\beta_{k_{S}}^{\mathrm{mdr}_{S}^{*}} F^{(\kappa)} e^{i \frac{\pi(\kappa+3)}{2(\kappa+1)} \operatorname{sign}\left(F^{(\kappa)}\right)}\right]}{\mid F^{(\kappa) \mid \frac{\kappa+3}{\kappa+1}}} & \text { if } v_{\theta_{j}}<\frac{H}{\Lambda} \frac{1}{x_{1}}
\end{array}\right], \\
\frac{1}{2} \frac{k_{1}}{k_{S}}\left(\frac{\Lambda}{H}\right)^{2}\left(\frac{k_{1}}{k_{S}} \frac{\Lambda}{H}\left(\mathcal{O}(1)+\frac{2}{3} \cos \left(\theta_{j}\right)\right) \operatorname{Im}\left[\beta_{k_{S}}^{\mathrm{mdr}^{*}}\right]+\cos \left(\theta_{j}\right) \operatorname{Re}\left[\beta_{k_{S}}^{\text {mdr }^{*}}\right]\right) & \text { if } \frac{\Lambda}{H} x_{1} \ll 1
\end{aligned},
$$


where we have again taken into account the averaging to zero of the strongly oscillating terms in final observables as the halo bias, and the $\mathcal{O}(1)$ term in the last line is specified in (117), appendix A.3. Equation (59) matches (57); to see that, note that in the modified initial state scenario, as we said, the contribution from the folded configuration corresponds to $\kappa \rightarrow \infty$, and that the dominating term in the second line of (57) is the one proportional to $\operatorname{Re}\left[\beta_{k_{S}}^{\mathrm{mis}}\right]$, since $\left|k_{1} \eta_{c} v_{\theta_{j}}\right| \ll 1$.

\subsection{General analysis for all cubic couplings in the effective action}

So far we have presented two examples to illustrate in details the kind of modifications that would occur in the squeezed limit of the bispectrum in the modified scenarios we consider. We now want to analyse the squeezed limit for all possible cubic coupling arising in a generic effective action for the inflaton.

Let us first summarize the key-points concerning the violation of the standard result for the bispectrum in the squeezed limit, which have emerged from the detailed examples discussed above:

- the most important modification to the standard result are due to the differences in the Whightman functions between the standard and the modified scenarios

- non-Gaussianities can be enhanced 15 in the squeezed limit by two effects:

- interference, which reduces the suppression due to the oscillating phase of the integrand in the time integral in the bispectrum formula, and is strongest when the perturbations depending on the large modes $k_{2,3} \sim k_{S}$ are in opposition of phase,

- accumulation in time, which leads to larger enhancement from the time integration when interactions scale with higher powers of $\frac{1}{a\left(\eta^{\prime}\right)} \sim-\eta^{\prime} 16$.

- if the squeezed mode and the time/scale of new physics are such that $\left|k_{1} \eta_{c}^{\left(k_{2,3}\right)}\right| \ll 1\left[17\right.$, or $\frac{k_{1}}{k_{S}} \frac{\Lambda}{H} \ll 1$, the perturbation depending on $k_{1}$ is already outside the horizon when the effects of new physics are relevant for the perturbations depending on $k_{2,3}$ (responsible for the maximum interference and enhancement, see previous point). Then the result is of the local form but with enhanced nonGaussianities (because of the particle content/creation, interference, and accumulation in time for $\left.\zeta_{k_{2}}, \zeta_{k_{3}}\right)$

- if instead $\left|k_{1} \eta_{c}^{\left(k_{2,3}\right)}\right| \gg 1$ or $\frac{k_{1}}{k_{S}} \frac{\Lambda}{H} \gg 1$ (in a realistic squeezed limit $k_{1} \neq 0$ ), the perturbation depending on $k_{1}$ is inside the horizon when the effects of the new physics on the $k_{2,3}$-perturbations are relevant and the bispectrum in the squeezed limit is not of the local form.

We want now to generalize our analysis beyond the two examples of interactions discussed above. It is convenient to adopt a different gauge than the one we have used so far: in the new gauge it is $\zeta=0$, and the perturbations are accounted for by a "matter field" perturbation $\varphi$. This gauge is fully equivalent, of course, to the one used before, and it is useful because it leads to a technically simpler expansion in perturbations [10]. It also makes clear from the outset the actual order in slowroll parameters of the cubic couplings, see [10. In particular, we can choose $\varphi$ to be the inflaton perturbation, expanding the inflaton in background plus perturbations

$$
\Phi(x, t)=\phi(t)+\varphi(x, t) .
$$

\footnotetext{
${ }^{15}$ The actual magnitude of the enhancements depends on the precise value of $\left|\beta_{k_{S}}\right|$ and the scales of "new" physics, and thus can only be estimated, as we will show in section 6 in our phenomenological approach.

${ }^{16}$ This is quite intuitive, due to the importance of early times (large $\left|\eta^{\prime}\right|$ ) around $\eta_{c}$ for the modified initial state scenario, or around the time of WKB violation/particle creation $\eta_{\mathrm{II}} \sim-\Lambda k_{S}^{-1} H^{-1}$ for the modified dispersion one. Indeed, if $\left|k_{1} \eta_{c} v_{\theta_{j}}\right| \ll 1$ or $\frac{k_{1}}{k_{S}} \frac{\Lambda}{H} \ll 1$, the integral for the bispectrum is approximately of the form $\int_{\eta_{c, \text { II }}}^{0} d \eta^{\prime} \eta^{\prime n} \sim \eta_{c, \text { II }}^{n+1}$, and one easily sees the growth with $n$. We will soon show a similar growth for $\left|k_{1} \eta_{c}\right| \gg 1, \frac{k_{1}}{k_{S}} \frac{\Lambda}{H} \gg 1$.

${ }^{17}$ We recall that the initial conditions for each mode of the perturbations are set when they are subhorizon, so that it is always $\left|k_{1} \eta_{c}^{\left(k_{1}\right)}\right| \gg 1$, see section 6
} 
The results in terms of the variable $\zeta$ are then obtained at the end by performing a gauge transformation as described in [10]:

$$
\zeta=-H \frac{\varphi}{\dot{\phi}}+\mathcal{O}\left(\epsilon^{2}, \varphi^{2}\right)
$$

so that

$$
\langle\zeta \zeta \zeta\rangle=-\frac{H^{3}}{\dot{\phi}^{3}}\langle\varphi \varphi \varphi\rangle+\left[\left\langle\mathcal{O}\left(\epsilon^{2}, \varphi^{2}\right) \varphi \varphi\right\rangle+\text { permutations }\right] .
$$

In particular, it is enough to consider the gauge-transformation at leading linear order, because the higher-order corrections will not yield time-integrated contributions to the bispectrum at tree-level, and, as it appears from the previous sections, it is the time-integrated contribution that leads to the leading modified results (see also [15, 20, 38]).

The most generic effective action for the inflaton $\Phi$ in single-field slow-roll inflation has been presented in 39 18 . In a Lorentz invariant theory, the terms in the action will be function of the Lorentz invariant objects $\Phi, g^{\mu \nu} \partial_{\mu} \Phi \partial_{\nu} \Phi, R^{\mu \nu} \partial_{\mu} \Phi \partial_{\nu} \Phi, \square \Phi, R$. In particular, those that are functions of $\Phi$ only, and not of the objects involving derivatives, constitute the scalar potential $V(\Phi)$. Recall also that all second order time derivatives (and first order derivatives of auxiliary fields) in higher order terms in the action (more than quadratic in the field) have to be eliminated using the equations of motion, see [39]. This applies for example to $\square \Phi$ and $R^{\mu \nu}$. In a Lorentz-broken theory (such as it is the case with modified dispersion relations), there are also additional terms that are not built up only from the Lorentz-invariant operators listed above. These terms involve higher derivatives of the fields that can be written as purely spatial derivatives in a convenient system of coordinates 19 .

By expanding the effective action in the inflaton perturbation (60), given the fundamental building blocks listed above (including the higher-derivative Lorentz-breaking ones), and using conformal time, the cubic couplings for the perturbation $\varphi$ have the most general and schematic form

$$
\int d \eta d^{3} x a^{4} \frac{\lambda_{n m s}}{\Lambda^{n+m+s-1}} \frac{\partial^{n} \varphi}{a^{n}} \frac{\partial^{m} \varphi}{a^{m}} \frac{\partial^{s} \varphi}{a^{s}} .
$$

Thus, a cubic coupling is individuated by the integers $n, m, s$ and by the coupling constant $\lambda_{n m s}$.

In equation (63) we have sloppily indicated both time and space derivatives (comoving coordinates) with the same symbol $\partial$ for notational simplicity and because it will simplify the counting of powers of momenta in the correlators. However, we will distinguish more carefully the two kinds of derivatives in the detailed calculations we are about to perform. We recall again, however, that in the case of time derivatives, their order is at most 1 (as we said, higher orders must be eliminated by using the equations of motion, see [39]). Finally, the dimensionless coefficients $\lambda_{n m s}$ can depend on $\dot{\phi}$ and in general on the slow-roll parameters, and are constrained by backreaction.

Let us comment on the possible values of $n, m, s$ for the cubic couplings (63). In a Lorentz-invariant theory, given the Lorentz-invariant objects listed above, $n, m, s$ can be at most equal to 120 , while in a Lorentz-broken theory their values can be higher (for the higher spatial derivatives couplings, in the convenient frame adapted to the Lorentz breaking).

Furthermore, observe that in a gauge-fixed theory, when solving for the gravitational constraints (for example those related to the lapse $N$ and shift-vector $N^{i}$ in the ADM formalism) there will also appear non-local operators. In the gauge we are using, they arise because of the solution 21

\footnotetext{
${ }^{18}$ Another way of writing the most generic effective action for a "matter scalar" takes advantage of the so-called Stueckelberg trick using the Goldstone mode for the broken time diffeomorphisms in the unitary gauge, see [70].

${ }^{19}$ Lorentz-breaking always implies a privileged frame, the convenient system of coordinates is precisely the one adapted to this frame.

${ }^{20}$ Obviously, integrating by parts, derivatives can be "moved around", in which case the more appropriate condition is that $\max (n+m+s)=3$. What we will say in the following is valid, of course, also for the terms obtained by integration by parts.

${ }^{21}$ The standard notation $(\vec{\partial})^{-2}$ for the inverse of $\sum_{j} \partial^{j} \partial_{j}$ is formal. It becomes clear after a Fourier transform.
} 
$N^{i} \simeq \epsilon^{\frac{1}{2}} \partial^{i}(\vec{\partial})^{-2} \dot{\varphi}+\mathcal{O}\left(\epsilon^{\frac{3}{2}}\right)$, see [10]. Thus, when expanding in perturbations, $n, m, s$ can assume value -1 because of the combination $(\vec{\partial})^{-2} \dot{\varphi}$ in the leading order contribution from $N^{i}$, relevant for the bispectrum. However, in the solution for $N^{i}$ this combination is acted upon by $\partial^{i}$, which increases by one the value of $n+m+s$ and thus "compensates" the -1 .

Moreover, $N^{i}$ enters the action only via the metric $g^{\mu \nu}$ or the extrinsic curvature. In the first case, the spatial index of $N^{i}$ will result always contracted with a spatial derivative, as it can be seen from $g^{\mu \nu} \partial_{\mu} \Phi \partial_{\nu} \Phi$ considering that the shift vector enters the components $g^{0 i}=N^{-2} N^{i}$ and $g^{i j}=N^{-2} N^{i} N^{j}$. Also when coming from the extrinsic curvature a component $N^{i}$ will always be accompanied by a spatial derivative, because the extrinsic curvature is defined as $K_{i j}=(2 N)^{-1}\left(\dot{h}_{i j}-2^{(3)} \nabla_{\{i} N_{j\}}\right)=$ $(2 N)^{-1}\left(\dot{h}_{i j}-h_{i k} \partial_{j} N^{k}-h_{j k} \partial_{i} N^{k}-N^{k} \partial_{k} h_{i j}\right)$, where $h_{i j},{ }^{(3)} \nabla$ are the ADM three-metric and relative covariant derivative. These properties imply that in a coupling where originally $N^{i}$ was present, the combination $\partial^{i}(\vec{\partial})^{-2} \dot{\varphi}$ will multiply at least one other factor with a spatial derivative, which increases further the value of $n+m+s$ by one.

Couplings with $n=m=s=0$ arise instead from the expansion of terms such as

$$
V(\Phi) \rightarrow \frac{V^{\prime \prime \prime}(\phi)}{3 !} \varphi^{3}, \quad \text { or } \quad\left(\frac{\partial^{r} \Phi}{a^{r}}\right)^{u} \Phi^{m} \rightarrow\left(\partial_{t}^{r} \phi\right)^{u} \phi^{m-3} \varphi^{3},
$$

and thus are higher-order in slow-roll and would give a negligible contribution to the bispectrum, see [10, 39, 70]. We will neglect them in the following. Moreover, couplings of the form $\frac{\lambda_{011}}{\Lambda} \varphi(\vec{\partial} \varphi)^{2}$, where $\vec{\partial}$ denotes spatial derivatives, are reabsorbed (eliminated) by field redefinitions such as $\varphi \rightarrow \varphi+\frac{\lambda_{011}}{2 \Lambda} \varphi^{2}$. Finally, couplings with a single time or spatial derivative are absent because of isotropy.

The two detailed examples of cubic couplings that we have analysed in sections 5.1, 5.2 fall into the scheme we are describing. Indeed, once written as in (63) using the relation between $\zeta$ and $\varphi$, they are of the type (see also the comment at the end of section 5.3.2)

$$
\begin{aligned}
\text { minimal cubic : } & \{n, m, s\}=\{1,1,-1\}, & \lambda_{11-1}^{\text {min cub }}=\sqrt{2 \epsilon} \frac{H}{M_{\text {Planck }}} \\
\text { quartic derivative : } & n=m=s=1, & \lambda_{111}^{\text {quart der }}=\sqrt{\frac{\epsilon}{2}} g \frac{H M_{\text {Planck }}}{\Lambda^{2}} .
\end{aligned}
$$

From equations (63), (61) and (4) the contribution to the bispectrum for a generic cubic coupling (63) reads

$$
\begin{array}{r}
\left\langle\zeta_{\vec{k}_{1}} \zeta_{\vec{k}_{2}} \zeta_{\vec{k}_{3}}\right\rangle_{\lambda} \propto \frac{\dot{\phi}^{3}}{H^{3}} \frac{\lambda_{n m s}}{\Lambda^{n+m+s-1}} \int_{\eta_{i}}^{0} d \eta^{\prime} a\left(\eta^{\prime}\right)^{4-n-m-s}\left(\partial^{n} G\right)_{k_{1}}\left(\partial^{m} G\right)_{k_{2}}\left(\partial^{s} G\right)_{k_{3}}+\text { permutations } \\
+ \text { complex conjugate }
\end{array}
$$

where we have neglected the momenta conserving delta function, $2 \pi$ factors and overall minus signs. We have used equation (61) to cast the result in terms of the perturbation $\zeta$ (the Whightman function in (67) are those of the $\zeta$-variable). $\left(\partial^{r} G\right)_{k}$ indicates the Fourier transform of $\partial^{r} G$.

We will now study the dominant contributions to (67) in the modified scenarios. We recall that, as it has emerged from the detailed examples, such contribution is due to the modification of the Whightman functions and occurs $i$ ) when the interference effects are stronger - that is, for $\zeta_{k_{2}}, \zeta_{k_{3}}$ in opposition of phase -, and ii) for the terms in the integrand with higher powers of $\eta^{\prime} \sim-\frac{1}{a}$ and, obviously, lower powers of $k_{1}$. In the following we will not pay attention to numerical factors of order $\mathcal{O}(1)$ in the formulas as they are irrelevant for our considerations.

In section (6), equipped with the results we are about to obtain, we will finally discuss which contributions, from all the possible couplings, determine the leading features of the bispectrum. 


\subsubsection{Modified initial state}

The relevant Whightman functions go as, see appendix A.2.

$$
\left(\partial_{\eta^{\prime}} G^{ \pm}\right)_{k}\left(0, \eta^{\prime}\right)= \pm \frac{H^{4}}{\dot{\phi}^{2}} k^{2} \eta^{\prime} \frac{e^{ \pm i k \eta^{\prime}}}{2 k^{3}}, \quad\left(\partial_{i}^{r \geq 0} G^{ \pm}\right)_{k}\left(0, \eta^{\prime}\right)= \pm \frac{H^{4}}{\dot{\phi}^{2}}\left( \pm i k_{i}\right)^{r}\left(1 \mp i k \eta^{\prime}\right) \frac{e^{ \pm i k \eta^{\prime}}}{2 k^{3}}
$$

so that when they are inserted in (67) we find the leading contribution linear in $\beta_{k_{i}}$

$$
\begin{array}{r}
\delta_{\beta}\left\langle\zeta_{\vec{k}_{1}} \zeta_{\vec{k}_{2}} \zeta_{\vec{k}_{3}}\right\rangle_{\lambda}^{\text {mis }} \propto \frac{\lambda_{n m s}}{\Lambda^{n+m+s-1}} \sum_{j=2}^{3} \beta_{k_{j}}^{*} \frac{H^{n+m+s+5}}{\dot{\phi}^{3} 2^{3} k_{1}^{3} k_{2}^{3} k_{3}^{3}} k_{a}^{n+1} k_{b}^{m+1} k_{c}^{s+1} \int_{\eta_{c}}^{\simeq 0} d \eta^{\prime}\left(-\eta^{\prime}\right)^{-1+n+m+s}\left(1+\left(-i k_{1} \eta^{\prime}\right)^{n_{t}^{(1)}-1}\right) \\
\times \frac{e^{i k_{1}\left(1+\cos \theta_{j}\right) \eta^{\prime}}}{2^{n_{t}^{(1)}}}+\text { hermitian, }
\end{array}
$$

where we have used equations (31), (32) and $a, b, c$ run over 1,2, 3 accounting for the permutations in (67), from which we take the dominant ones 22. Finally, $n_{t}^{(1)}=\{0,1\}$ accounts for the presence or not of time derivatives acting on $G\left(k_{1}, \eta^{\prime}\right)$.

We concentrate now on the time-integral, which can be computed in closed form. By writing $v \equiv n+m+s\left(+n_{t}^{(1)}-1\right)$, the integral is well defined for the values attainable by $n, m, s$, discussed in section 5.3 , and gives $(j=2,3)$

$$
\begin{aligned}
\int_{\eta_{c}}^{0} d \eta^{\prime} \eta^{\prime-1+v} e^{i k_{1}\left(1+\cos \theta_{j}\right) \eta^{\prime}} & =\frac{(-1)^{v}}{\left(i k_{1}\right)^{v}}\left(\sum_{r=0}^{v-1}\left(\begin{array}{c}
v-1 \\
r
\end{array}\right) \frac{r !\left(-i k_{1} \eta_{c}\right)^{v-1-r}}{\left(1+\cos \theta_{j}\right)^{r+1}} e^{i k_{1} \eta_{c}\left(1+\cos \theta_{j}\right)}-\frac{(v-1) !}{\left(1+\cos \theta_{j}\right)^{v}}\right) \\
& \approx \begin{cases}-\frac{\eta_{c}^{v}}{v} & \text { if }\left|k_{1} \eta_{c}\left(1+\cos \theta_{j}\right)\right| \ll 1 \\
\frac{(-1)^{v-1}}{\left(i k_{1}\right)^{v}} \frac{(v-1) !}{\left(1+\cos \theta_{j}\right)^{v}} & \text { if }\left|k_{1} \eta_{c}\left(1+\cos \theta_{j}\right)\right| \gg 1,\end{cases}
\end{aligned}
$$

where in the last passage we have written the leading contribution, also considering the averaging to zero of large oscillations in the final observables as the halo bias.

Inserting this result in equation (69), we obtain the leading contribution (reinstating the delta function from conservation of momentum)

$$
\delta_{\beta}\left\langle\zeta_{\vec{k}_{1}} \zeta_{\vec{k}_{2}} \zeta_{\vec{k}_{3}}\right\rangle_{\lambda}^{\mathrm{mis}}=(2 \pi)^{3} \delta^{(3)}\left(\sum_{i} \vec{k}_{i}\right) \mathcal{B}_{\lambda_{\mathrm{nms}}}^{\mathrm{mis}} P_{\mathrm{st}}\left(k_{1}\right) P_{\mathrm{st}}\left(k_{S}\right)
$$

where, having substituted back $n+m+s$ in place of $v$,

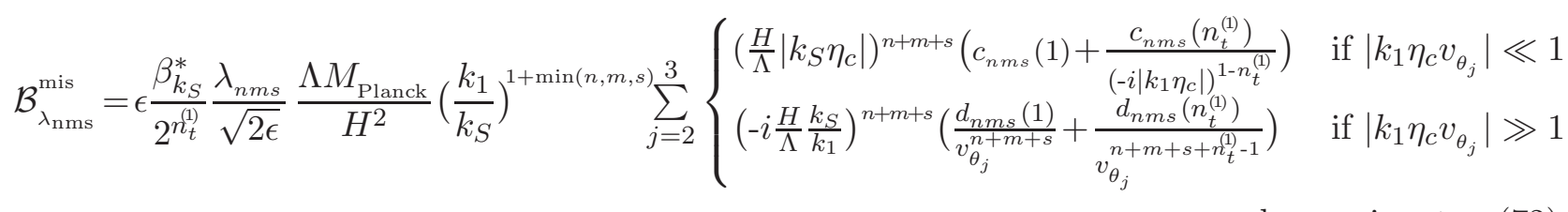

$$
\begin{aligned}
& + \text { complex conjugate }
\end{aligned}
$$

with

$$
v_{\theta_{j}} \equiv\left(1+\cos \theta_{j}\right), \quad c_{n m s}(x) \equiv \frac{c_{\lambda}}{(n+m+s-1+x)}, \quad d_{n m s}(x) \equiv(-1)^{x-1}(n+m+s-2+x) ! d_{\lambda},
$$

${ }^{22}$ Recall that, since we are considering only scalar perturbations, after the expansion the spatial derivatives indexes are contracted by the background metric, leading thus to contributions proportional, by permutation, to $\vec{k}_{1} \cdot \vec{k}_{2}, \vec{k}_{1} \cdot \vec{k}_{3}$, $\vec{k}_{2} \cdot \vec{k}_{3}$. But then, in the squeezed limit, the contributions proportional to $\vec{k}_{1} \cdot \vec{k}_{2}$ and $\vec{k}_{1} \cdot \vec{k}_{3}$ give an overall contribution proportional to $\vec{k}_{1} \cdot\left(\vec{k}_{2}+\vec{k}_{3}\right) \sim k_{1}^{2}\left[12\right.$ that is subdominant with respect to the contribution proportional to $\vec{k}_{2} \cdot \vec{k}_{3}$, which goes as $\vec{k}_{2} \cdot \vec{k}_{3} \rightarrow-k_{2} k_{3}=-k_{S}^{2}$ in the limit. The latter is thus the leading one that we consider here and in the following. 
and $c_{\lambda}, d_{\lambda}$ are numerical factors of order one, possibly depending on $\theta_{j}$.

We observe that the generic contributions present both enhancement factors (such as $\left|k_{S} \eta_{c}\right| \gg 1$, or $\frac{k_{S}}{k_{1}} \gg 1$ ) as well as suppressing ones (such as $\left(\frac{H}{\Lambda}\right)^{n+m+s-1}$ and $\lambda_{n m s}$ ). We also observe that for $\left|k_{1} \eta_{c} v_{\theta_{j}}\right| \gg 1$ the contribution will not be in general of the local form. However, such contributions could be suppressed and thus subleading. We will discuss these points in details in section 6. As a check, note that when specialized to the couplings (65), (66) that we studied in details previously, equation (172) leads to the results (37), (57) that we found before, with the proper $c_{\lambda}, d_{\lambda}$.

\subsubsection{Modified dispersion relations}

As discussed in section 5.1 .3 the leading contribution to the bispectrum arises when the Whightman functions have support mostly in the interval of times in regions IV/III, given the solution (11). They go as, see appendix A.2.

$$
\left(\partial_{\eta^{\prime}} G^{ \pm}\right)_{k}\left(0, \eta^{\prime}\right)=-i \frac{H^{3}}{\dot{\phi}^{2}} k \frac{\gamma^{(*)}\left(k, \eta^{\prime}\right)}{a\left(\eta^{\prime}\right)} \frac{e^{ \pm i \frac{\Lambda}{H} \Omega\left(k, \eta^{\prime}\right)}}{2 k^{2}}, \quad\left(\partial_{i}^{r \geq 0} G^{ \pm}\right)_{k}\left(0, \eta^{\prime}\right)=\frac{H^{3}}{\dot{\phi}^{2}}\left( \pm i k_{i}\right)^{r} \frac{\chi^{(*)}\left(k, \eta^{\prime}\right)}{a\left(\eta^{\prime}\right)} \frac{e^{ \pm i \frac{\Lambda}{H} \Omega\left(k, \eta^{\prime}\right)}}{2 k^{2}},
$$

where $\chi^{(*)}\left(k, \eta^{\prime}\right)$ is defined in equation (108) and $\gamma^{(*)}\left(k, \eta^{\prime}\right)$ in (109).

Once again, it is convenient to write the contribution to the bispectrum in terms of the variables defined in equation (38). Inserting the Whightman functions in (67), the leading contribution linear in $\beta_{k_{i}}$ reads

$$
\begin{aligned}
\delta_{\beta}\left\langle\zeta_{\vec{k}_{1}} \zeta_{\vec{k}_{2}} \zeta_{\vec{k}_{3}}\right\rangle_{\lambda}^{\mathrm{mdr}} \propto \frac{\lambda_{n m s}}{\Lambda^{n+m+s-1}} \sum_{j=2}^{3} \beta_{k_{j}}^{*} \frac{H^{n+m+s+5}}{\dot{\phi}^{3} 2^{3} k_{1}^{3} k_{2}^{3} k_{3}^{3}}\left(\frac{\Lambda}{H}\right)^{n+m+s} \frac{k_{a}^{n+1} k_{b}^{m+1} k_{c}^{s+1}}{k_{S}^{n+m+s}} \\
\times \int_{y_{\mathrm{II}} \sim-1}^{y \lesssim-\frac{H}{\Lambda}} d y^{\prime}\left(-y^{\prime}\right)^{n+m+s-1} \prod_{h=\{a, b, c\}}\left(i \gamma^{(*)}\left(x_{h}, y^{\prime}\right)\right)^{n_{t}^{(h)}}\left(\chi^{(*)}\left(x_{1}, y^{\prime}\right)\right)^{1-n_{t}^{(1)}} e^{i \frac{\Lambda}{H} x_{1} \tilde{v}_{\theta_{j}}^{(n)}\left(y^{\prime}\right)}+\text { hermitian},
\end{aligned}
$$

where we have used equation (42), $n_{t}^{(a, b, c)}=\{0,1\}$ accounts for the presence or not of time derivatives in the coupling acting on $\zeta_{k_{a, b}, c}$, and $a, b, c$ run over 1,2,3 taking into account the permutations, from which we take the dominant ones according to footnote (22). Depending on the specific Lorentz-broken model, $n, m, s$ can assume values larger than 1 such that $n+m+s$ can be larger than 323 .

The solution for the integral can be again obtained by asymptotic techniques as those employed in sections 5.1.3, 5.2.2, so that the leading contribution reads

$$
\delta_{\beta}\left\langle\zeta_{\vec{k}_{1}} \zeta_{\vec{k}_{2}} \zeta_{\vec{k}_{3}}\right\rangle_{\lambda}^{\mathrm{mdr}}=(2 \pi)^{3} \delta^{(3)}\left(\sum_{i} \vec{k}_{i}\right) \mathcal{B}_{\lambda_{\mathrm{nms}}}^{\mathrm{mdr}} P_{\mathrm{st}}\left(k_{1}\right) P_{\mathrm{st}}\left(k_{S}\right)
$$

where, having written $n+m+s=v$ and $n+m+s+n_{t}^{(1)}-1=v_{t}$, and used table 1 in appendix A.3,

$$
\begin{aligned}
& \mathcal{B}_{\lambda_{\mathrm{nms}}}^{\mathrm{mdr}}=\epsilon \beta_{k_{S}}^{*} \frac{\lambda_{n m s}}{\sqrt{2 \epsilon}} \frac{\Lambda M_{\text {Planck }}}{H^{2}}\left(\frac{k_{1}}{k_{S}}\right)^{1+\min (n, m, s)} i_{t}^{n_{t}^{(1)}}
\end{aligned}
$$

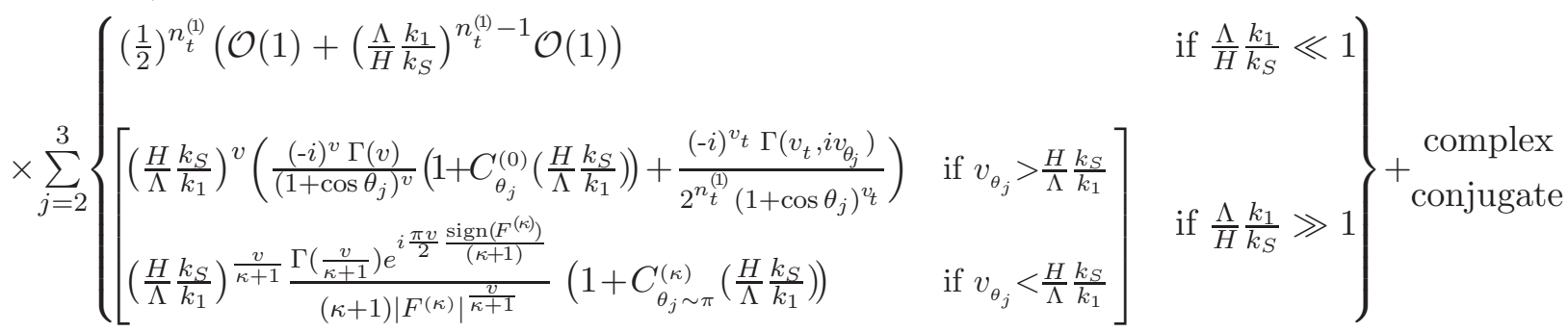

\footnotetext{
${ }^{23}$ However, time derivatives are at most of order 1 , see section 5.3 and 39 .
} 
with

$$
C_{\theta_{j}}^{(\rho)}\left(\frac{H}{\Lambda} \frac{k_{S}}{k_{1}}\right) \equiv \sum_{r, n=0}^{\infty} c_{\theta_{j}}^{r, n}\left(\frac{H}{\Lambda} \frac{k_{S}}{k_{1}}\right)^{\frac{\kappa+r+n}{\rho+1}} \frac{\Gamma\left(\frac{v+\kappa+r+n}{\rho+1}\right) e^{i \frac{\pi(\kappa+r+n)}{(\rho+1)} \frac{\operatorname{sign}\left(F^{(\rho)}\right)}{2}}}{\Gamma\left(\frac{v}{\rho+1}\right)\left|F^{(\rho+n)}\right|^{\frac{\kappa+r+n}{\rho+1}}}
$$

containing all the subleading asymptotic contributions (the $c_{\theta_{j}}^{r, n}$ are calculable order one coefficients, whose precise form is irrelevant for us, obtained from the expansion of the integrand in (75)). Finally, $v_{\theta_{j}}$ has been defined in (32),$\Gamma\left(v_{t}, i v_{\theta_{j}}\right)$ is the lower incomplete gamma function, and the $\mathcal{O}(1)$ factors in the second line of (77) are written in details in (118), appendix A.3.

This result is very similar to that obtained in the modified initial state scenario, except for two differences. One is that now $n+m+s$ can also assume values larger than 3 in the case of couplings deriving from the Lorentz-breaking terms in the action. The other is that for (nearly) folded configurations $\kappa$ is finite and determined by the first correction to the standard dispersion relations (see (45)), whereas in the modified initial state scenario it is $\kappa \rightarrow \infty$ for the folded configuration. A part from this, the similar structure of the two results can again be seen as showing that indeed the squeezed limit is dominated, at leading order, by the generic features common to both scenarios (such as particle content/creation, interference and time accumulation).

As a check, one finds that when equation (777) is specialized to the couplings (65), (66) that we studied previously as detailed examples, it leads to the results we have found before, see (47), (59). Note, in particular, that since the one in equation (48) is the sum of two elementary cubic couplings (one with only time derivatives, the other with one time and two space derivatives) such that for the folded configurations the leading contribution is cancelled out, for those configurations the result (59) is given by the first subleading correction in equation (77).

\section{Signatures of very high energy physics in the squeezed limit}

In the previous sections we have studied the contributions to the bispectrum in the squeezed limit in scenarios with modified initial state or modified dispersion relations at high energies. This has lead to the formulas (71)-(72) and (76)-(77) for the leading corrections to the standard result for all possible cubic couplings, see (63), in the effective Lagrangian.

Armed with these results, we are now going to

- discuss the leading features of the contributions to the bispectrum in the squeezed limit, individuating also which cubic couplings yield the largest contributions;

- obtain the specific predictions for the modified scenarios reviewed in section 3

Concerning the analysis of the leading features of the bispectrum in the squeezed limit, we will focus on the dependence on the probed large scale $k_{1}^{-1}$ and on the magnitude of the non-Gaussianities. Indeed, those are the most interesting features for observational purposes (for example regarding the halo bias) [13, 14].

The results(71)-(72) and (76)-(77) show that the bispectrum has different kinds of behaviour subject to the interplay between the squeezed momentum scale $k_{1}$ and the scales/times $\Lambda / \eta_{c}$ of new physics. In particular, the different behaviours occur depending on whether $\left|k_{1} \eta_{c}^{\left(k_{S}\right)}\right|$ or $\frac{k_{1}}{k_{S}} \frac{\Lambda}{H}$ are larger or smaller than 1 . We will study the various cases separately.

\subsection{Enhancements}

We investigate here the possibility of enhancements. As we have adopted a phenomenological approach, we do not have the full-detailed knowledge of the magnitude of the Bogoliubov coefficient $\beta_{k_{S}}$, which the leading contributions to the bispectrum depend upon, see (71)-(72) and (76)-(777). However, we 
know the constraints that it has to satisfy for phenomenological reasons and self-consistency of the theory (see section 3.4). Therefore we can estimate the largest possible enhancements.

Also, it is noteworthy that the leading contributions depend only on $\beta_{k_{S}}$, which is independent of $k_{1}$, see (72), (77). This means that our ignorance of the specific form of $\beta_{k_{S}}$ does not affect our knowledge of the $k_{1}$-dependence of the leading contributions.

We turn now to the detailed analysis.

Cases $\left|k_{1} \eta_{c}^{\left(k_{s}\right)}\right| \ll 1$ and $\frac{k_{1}}{k_{S}} \frac{\Lambda}{H} \ll 1$

In these cases the corrections to the bispectrum (71)-(72), (76)-(77) for a generic cubic coupling contribute a parameter $24 f_{N L} \sim \mathcal{B}_{\lambda_{\mathrm{nms}}}^{\mathrm{mis} \text { mdr }}$, where, at leading order,

$$
\begin{aligned}
\left|\mathcal{B}_{\lambda_{\mathrm{nms}}}^{\mathrm{mis}, \mathrm{mdr}}\right| & \sim \epsilon\left|\beta_{k_{S}}\right| \frac{\lambda_{n m s}}{\sqrt{2 \epsilon}} \frac{\Lambda M_{\text {Planck }}}{H^{2}}\left(\frac{k_{1}}{k_{S}}\right)^{1+\min (n, m, s)}\left(\frac{H D}{\Lambda}\right)^{n+m+s}\left(1+\left(\frac{k_{1} D}{k_{S}}\right)^{n_{t}^{(1)}-1} \mathcal{O}(1)\right) \\
& \leq \epsilon \sqrt{|\mu|} \frac{\lambda_{n m s}}{\sqrt{2}} \frac{M_{\text {Planck }}^{2}}{H \Lambda}\left(\frac{k_{1}}{k_{S}}\right)^{1+\min (n, m, s)}\left(\frac{H D}{\Lambda}\right)^{n+m+s}\left(1+\left(\frac{k_{1} D}{k_{S}}\right)^{n_{t}^{(1)}-1} \mathcal{O}(1)\right)
\end{aligned}
$$

and where $D=\left|k_{S} \eta_{c}^{\left(k_{S}\right)}\right|$ in the modified initial state case, while $D=\frac{\Lambda}{H}$ in the scenario with modified dispersion relation 25. For the final inequality (80) we have used the constraint (17) on $\left|\beta_{k_{S}}\right|$.

Writing the above expressions using a common symbol $D$ for both kinds of modified scenarios is useful to make evident the similarity of the results in the two different cases. As we remarked, this similarity is an indication that the squeezed limit is dominated by the generic features common to both of them (particle content/creation, interference effects).

We analyse now in details the features of (80), paying particular attention to the possibility of enhancements (we will focus on the scale dependence in section 6.2).

If we consider first $D=\left|k_{S} \eta_{c}^{\left(k_{S}\right)}\right|$ (modified initial state), the inequality (80) reads

$$
\left|\mathcal{B}_{\lambda_{\mathrm{nms}}}^{D=\left|k_{S} \eta_{c}\right|}\right| \leq \epsilon \sqrt{|\mu|} \frac{\lambda_{n m s}}{\sqrt{2}} \frac{M_{\text {Planck }}^{2}}{H \Lambda}\left(\frac{k_{1}}{k_{S}}\right)^{1+\min (n, m, s)}\left(\frac{\left|p_{S}\left(\eta_{c}\right)\right|}{\Lambda}\right)^{n+m+s}\left(1+\left(\frac{\left|p_{1}\left(\eta_{c}\right)\right|}{H}\right)^{n_{t}^{(1)}-1} \mathcal{O}(1)\right),
$$

where $p_{S(1)}\left(\eta_{c}\right) \equiv-k_{S(1)} \eta_{c}^{\left(k_{S}\right)} H$ is the physical momentum associated with the wavenumber $k_{S(1)}$ at the boundary time $\eta_{c}^{\left(k_{S}\right)}$.

When instead $D=\frac{\Lambda}{H}$, such as in the cases of modified dispersion relations or NPHS initial state, the expression (80) simplifies and we obtain

$$
\left|\mathcal{B}_{\lambda_{\mathrm{nm}} \mathrm{NPHS}, \mathrm{mdr}}\right| \leq \epsilon \sqrt{|\mu|} \frac{\lambda_{n m s}}{\sqrt{2}} \frac{M_{\text {Planck }}^{2}}{H \Lambda}\left(\frac{k_{1}}{k_{S}}\right)^{1+\min (n, m, s)}\left(1+\left(\frac{k_{1}}{k_{S}} \frac{\Lambda}{H}\right)^{n_{t}^{(1)}-1} \mathcal{O}(1)\right) .
$$

From (81), (82), we can understand various characteristics of the amplitude of the non-Gaussianities when $\left|k_{1} \eta_{c}^{\left(k_{S}\right)}\right|, \frac{k_{1}}{k_{S}} \frac{\Lambda}{H} \ll 1$. First of all, we see that (81) is maximized for $p_{S}\left(\eta_{c}\right)=\Lambda$, that is, when the physical momentum associated with $k_{S}$ at $\eta_{c}^{\left(k_{S}\right)}$ is really at the largest scale that we can trust our effective theory at: the scale of new physics $\Lambda$. This is precisely what occurs in the NPHS scenario of initial state modification: the initial condition at $\eta_{c}^{\left(k_{S}\right)}$ is fixed by construction when the physical momentum is at the cutoff scale $\Lambda$, and we get (82).

In the case of modified dispersion relations, equation (82) can be re-written in a way that makes the similarity with the modified initial case even more evident. It is straightforward to obtain an equation which is analogous to (81), but where the place of $p_{S}\left(\eta_{c}\right)$ is taken by the physical momentum

\footnotetext{
${ }^{24}$ As before, 'mis' indicates the modified initial state scenarios, while 'mdr' those with modified dispersion relations.

${ }^{25}$ However, in the NPHS approach $\left|k_{S} \eta_{c}^{\left(k_{S}\right)}\right|=\frac{\Lambda}{H}$.
} 
$p_{S}\left(\eta_{\text {II }}^{\left(k_{S}\right)}\right)$ associated with $k_{S}$ at the particle creation/WKB breaking time $\eta_{\text {II }}^{\left(k_{S}\right)}$, which indeed has a magnitude of the order of $\Lambda$, see (82). This very simple exercise of rewriting is not useless, as it is another indication of the universality of the features of the squeezed limit in the modified scenarios.

Coming back to the question whether there can be actual overall enhancements, potentially interesting for observations, one needs to take into account all the factors in the equations (81), (82) for $\mathcal{B}_{\lambda}^{\text {mis, mdr }}$ : both the enhancing $\left(\frac{M_{\mathrm{Planck}}^{2}}{H \Lambda}\right)$ and the suppressing $\left(\lambda_{n m s} \epsilon \sqrt{|\mu|}\left(\frac{k_{1}}{k_{S}}\right)^{1+\min (n, m, s)}\right)$. As for the latter ones, it is immediately evident that terms with higher number of derivatives are more suppressed since $\min (n, m, s)$ will be higher. This tells us that the contributions from the higher-derivative Lorentz-breaking couplings in the scenarios with modified dispersion relations, where $n, m, s$ can assume values also much larger that one, will be suppressed.

Considering then the possible values of $n, m, s$, see section 5.3 , it turns out, as it was in fact imaginable, that the cubic couplings (18) and (48) that we have discussed as detailed examples yield (among) the least suppressed contributions. Indeed, they lead to a suppression respectively of order $\left(\frac{k_{1}}{k_{S}}\right)^{0}$ and $\left(\frac{k_{1}}{k_{S}}\right)^{2}$, see (65), (66). In particular, the minimal cubic coupling (65) is not actually suppressed by factors $\frac{k_{1}}{k_{S}}$. In fact, that coupling is leading also in the standard scenario, see [12].

If we concentrate on these dominant (less suppressed) couplings, which we had studied in details as examples in sections 5.1 and 5.2 , we find that there can indeed be actual overall enhancement of the non-Gaussianities with respect to the standard scenario for certain values of the scale of new physics. Let us discuss a concrete example: take $\epsilon \sim|\mu| \sim 10^{-2}, H \sim 10^{-5} M_{\text {Planck }}$, compatible with the results of WMAP, and $\Lambda \sim 10^{-3} M_{\text {Planck }}$, which corresponds to the supersymmetric GUT scale. Then, from (82), if we can probe down to $\frac{k_{1}}{k_{S}} \lesssim 10^{-2}$, the largest possible enhanced amplitude that we could observe would be roughly of the order of

$$
\left|\mathcal{B}_{\text {min cub }}^{\mathrm{NPHS}, \mathrm{mdr}}\right| \sim 10 \epsilon \sim 10\left(1-n_{s}\right) .
$$

in the case of the minimal coupling (65), while it will be

$$
\left|\mathcal{B}_{\text {quart der }}^{\mathrm{NPHS} \text { mdr }}\right| \sim 10^{3} g\left(1-n_{s}\right) \sim 10\left(1-n_{s}\right)
$$

in the case of the quartic derivative interaction (666) with $g \sim 10^{-2}$.

It is noteworthy that for the higher (quartic)-derivative interaction the additional suppressing factor $\left(\frac{k_{1}}{k_{S}}\right)^{2}$ and enhancing factor $\frac{M_{\text {Planck }}^{2}}{\Lambda^{2}}$ (essentially due to the power of $a^{-1}$ scaling) compensate each other to give a result of the same magnitude as that of the minimal cubic coupling. This result is quite different from the one in the standard scenario, where the $a^{-1}$ scaling of the interactions does not have any important effect since the time integral in the bispectrum is dominated by late times.

Having obtained these field theory results, one should study the implications for the actual experiments. This goes beyond the scope of this work, and we leave it for future research. However, we observe that, according to the analysis of [12, values as $\frac{k_{1}}{k_{S}} \sim 10^{-2}$ are indeed within the reach of LSS investigations, such as for example EUCLID, which in the most optimistic estimates can probe down to $\frac{k_{1}}{k_{S}}<10^{-3}[12]$.

\section{Cases $\left|k_{1} \eta_{c}^{\left(k_{s}\right)}\right| \gg 1$ and $\frac{k_{1}}{k_{S}} \frac{\Lambda}{H} \gg 1$}

In this case the correction (71)-(72) , (76)-(77) to the bispectrum for a generic cubic coupling contributes a parameter $f_{N L} \sim \mathcal{B}_{\lambda_{\text {nms }}}^{\text {mis, mdr }}$ with, at leading order,

$$
\begin{aligned}
\left|\mathcal{B}_{\lambda_{\mathrm{nms}} \mathrm{mis, \textrm {mdr }}}\right| & \sim \epsilon\left|\beta_{k_{S}}\right| \frac{\lambda_{n m s}}{\sqrt{2 \epsilon}} \frac{\Lambda M_{\text {Planck }}}{H^{2}}\left(\frac{k_{1}}{k_{S}}\right)^{1+\min (n, m, s)} E^{(n, m, s)}\left(\frac{H}{\Lambda} \frac{k_{S}}{k_{1}}\right) \\
& \leq \epsilon \sqrt{|\mu|} \frac{\lambda_{n m s}}{\sqrt{2}} \frac{M_{\text {Planck }}^{2}}{H \Lambda}\left(\frac{k_{1}}{k_{S}}\right)^{1+\min (n, m, s)} E^{(n, m, s)}\left(\frac{H}{\Lambda} \frac{k_{S}}{k_{1}}\right)
\end{aligned}
$$


where in the last inequality we have used the constraint (17) on $\left|\beta_{k_{S}}\right|$. Here,

$$
E^{(n, m, s)}\left(\frac{H}{\Lambda} \frac{k_{S}}{k_{1}}\right)=\sum_{j=2}^{3}\left\{\begin{array}{lr}
\left(\frac{H}{\Lambda} \frac{k_{S}}{k_{1}}\right)^{n+m+s}\left(\mathcal{O}(1)+\sum_{r=0}^{\infty} \mathcal{O}\left(\left(\frac{H}{\Lambda} \frac{k_{S}}{k_{1}}\right)^{\kappa+r}\right)\right)_{\left[\theta_{j}\right]} & \text { if } 1+\cos \left(\theta_{j}\right)>\frac{H}{\Lambda} \frac{k_{S}}{k_{1}} \\
{\left[\begin{array}{ll}
\left(\frac{H D}{\Lambda}\right)^{n+m+s}\left(1+\left(\frac{k_{1} D}{k_{S}}\right)^{n_{t}^{(1)}-1} \mathcal{O}(1)\right)_{\left[\theta_{j} \sim \pi\right]} & \operatorname{mis} \\
\left(\frac{H}{\Lambda} \frac{k_{S}}{k_{1}}\right)^{\frac{n+m+s}{\kappa+1}}\left(1+\sum_{r=0}^{\infty} \mathcal{O}\left(\left(\frac{H}{\Lambda} \frac{k_{S}}{k_{1}}\right)^{\frac{\kappa+r}{k+1}}\right)\right)_{\left[\theta_{j} \sim \pi\right]} & \operatorname{mdr}
\end{array}\right]} & \text { if } 1+\cos \left(\theta_{j}\right)<\frac{H}{\Lambda} \frac{k_{S}}{k_{1}}
\end{array},\right.
$$

where we have emphasized the orders of magnitude, as we are interested in the enhancements -for the detailed expressions see (72), (177) - (the $\theta_{j}$ dependence is indicated by the label $\left[\theta_{j}\right]$ ).

A few features are evident at first sight from (86)), (87). First of all, the powers of $\left(\frac{H}{\Lambda} \frac{k_{S}}{k_{1}}\right)$ in equations (86), (87) are suppressing factors, because we are considering the case $\frac{k_{1}}{k_{S}} \frac{\Lambda}{H} \gg 1$.

Second, the (nearly) folded configurations (singled out by the condition $1+\cos \left(\theta_{j}\right)<\frac{H}{\Lambda} \frac{k_{S}}{k_{1}} \ll 1$ ) are enhanced with respect to the other configurations ( $D$ has been defined below equation (79) ), although less so in the scenario of modified dispersion relations. It follows, as we see from (87), that the result has a non-trivial $\theta_{j}$ dependence (different enhancements for different values of $\theta_{j}$, recall also that we consider a realistic squeezed limit where $k_{1}$ is small but nonzero).

Finally, we see that the higher the number of derivatives, the more the suppression because of the factors in (86)), (87). In this respect, considering the possible values of $n, m, s$, the cubic couplings (18) and (48) (see their schematic form in (65), (66)), which we have discussed as detailed examples, turn out again to be the most dominant ones.

Equation (86) also includes enhancement factors such as $\frac{M_{\text {Planck }}^{2}}{H \Lambda}$, therefore the presence of actual overall enhancements depends once more on the values of the scales and quantities at play. To give some pointers about the possibilities, let us again consider the same example as before and the couplings (18) and (48), summarized in (65), (66). Recall that we take $\epsilon \sim|\mu| \sim 10^{-2}, H \sim 10^{-5} M_{\text {Planck }}$, compatible with the results of WMAP, and $\Lambda \sim 10^{-3} M_{\text {Planck }}$ (supersymmetric GUT scale).

Then, if we can only probe values down to $\frac{k_{1}}{k_{S}} \gtrsim 10^{-2}$, we are in the case $\frac{k_{1}}{k_{S}} \frac{\Lambda}{H}>1$ and from (866), (87), (65) we find the bound, roughly,

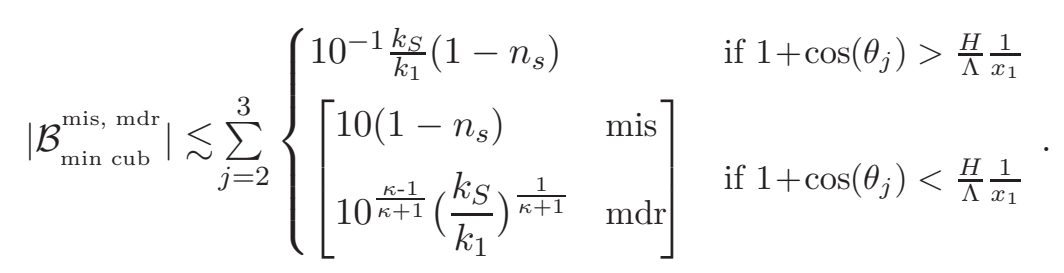

We see that for $\frac{k_{1}}{k_{S}} \simeq 10^{-2}$ the folded configurations, both in the modified initial state and modified dispersion relations scenarios, could be ten times enhanced with respect to the standard result, whereas the other configurations would present only a moderate enhancement compared to the result in the standard scenario.

In the case of the quartic-derivative interaction (48), (66), for a coupling $g \sim 10^{-2}$, we find from (72), (777) - taking into account the comment at the end of section 5.3.2-, or directly from (57), (59.) that

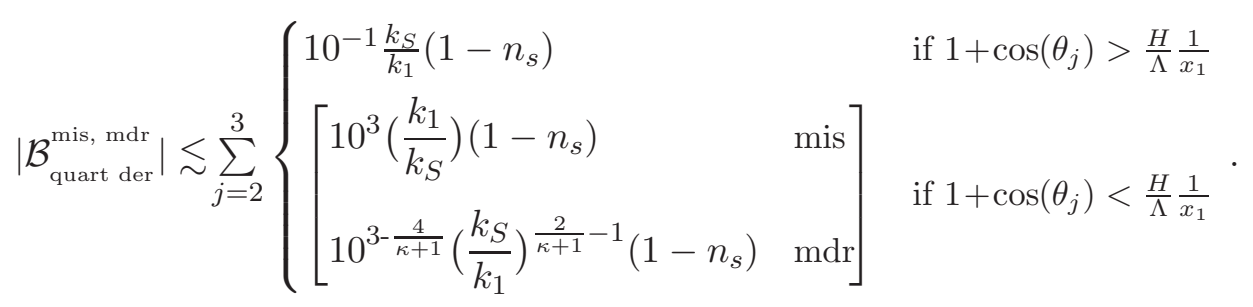

Note that for $\frac{k_{1}}{k_{S}} \simeq 10^{-2}$, (88) and (89) are of the same magnitude, which again shows that in the modified scenarios the different $a^{-1}$ scaling of the higher-derivative interactions can compensate for the 
stronger suppression by higher powers of $\frac{k_{1}}{k_{S}}$ and make the squeezed limit sensitive to higher-derivative interactions.

\subsection{Dependence on $k_{1}$}

We discuss now the dependence on the squeezed wavenumber $k_{1}$. Recall that the leading contributions depend only on $\beta_{k_{S}}$, which is independent of $k_{1}$, see (71)-(172) and (76)-(77). Thus, the $k_{1}$-dependence of the leading corrections to the bispectrum in the squeezed limit is not affected by our ignorance of the specific form of $\beta_{k_{S}}$.

In the following, we will rewrite equations (71)-(72) and (76)-(77) highlighting the dependence on $k_{1}$. We will neglect the momenta conserving delta function to avoid cluttering formulas. Again, we find that the field theoretical results for the modified scenarios have two different kinds of behaviour, depending on the magnitude of the scale of new physics as compared to the sensitivity of the observations (smallest $k_{1}$ that can be probed). We will stress the differences with respect to the standard scenario, where the bispectrum grows in the squeezed limit as $\sim k_{1}^{-3}$ [10 12], and also compare to the result obtained with the approximated template proposed in [15] for scenarios with modified initial state, which leads to a bispectrum growing as $\sim k_{1}^{-2}$, see [13, 14].

Cases $\left|k_{1} \eta_{c}^{\left(k_{s}\right)}\right| \ll 1$ and $\frac{k_{1}}{k_{S}} \frac{\Lambda}{H} \ll 1$

In this cases, equations (71)-(72) and (76)-(77) yield at leading order

$\delta_{\beta}\left\langle\zeta_{\vec{k}_{1}}(\eta) \zeta_{\vec{k}_{2}}(\eta) \zeta_{\vec{k}_{3}}(\eta)\right\rangle_{\lambda_{\mathrm{nms}}}=\epsilon\left|\beta_{k_{S}}\right| \frac{\lambda_{n m s}}{\sqrt{2 \epsilon}} \frac{\Lambda M_{\mathrm{Planck}}}{H^{2}}\left(\frac{H D}{\Lambda}\right)^{n+m+s}\left(\frac{k_{1}}{k_{S}}\right)^{1+\min (n, m, s)}\left(1+c_{n_{t}^{(1)}}\left(\frac{k_{1} D}{k_{S}}\right)^{n_{t}^{(1)}-1}\right) P_{\mathrm{st}}\left(k_{1}\right) P_{\mathrm{st}}\left(k_{S}\right)$,

where, as before, $D=\left|k_{S} \eta_{c}^{\left(k_{s}\right)}\right|$ or $\frac{\Lambda}{H}$, respectively for modified initial state or modified dispersion relations.

The corrections (90) scale as $k_{1}^{-3+\min (n, m, s)+n_{t}^{(1)}}$ (recall $n_{t}^{(1)}=\{0,1\}$, defined below equations (69)), (75)). We thus find that higher-derivative couplings (larger values for $n, m, s$ ) grow more slowly for small $k_{1}$. The contribution that grows the fastest is of the form

$$
\delta_{\beta}\left\langle\zeta_{\vec{k}_{1}}(\eta) \zeta_{\vec{k}_{2}}(\eta) \zeta_{\vec{k}_{3}}(\eta)\right\rangle_{\min (n, m, s)=-1}=\epsilon\left|\beta_{k_{S}}\right| \frac{\lambda_{n m s}}{\sqrt{2 \epsilon}} \frac{\Lambda M_{\text {Planck }}}{H^{2}}\left(\frac{H D}{\Lambda}\right)^{n+m+s} P_{\mathrm{st}}\left(k_{1}\right) P_{\mathrm{st}}\left(k_{S}\right),
$$

that is, it has a local shape $k_{1}^{-3}$, and can be found for couplings such as the minimal coupling cubic, where $\min (n, m, s)=-1$ (recall that this value occurs due to the combination $(\vec{\partial})^{-2} \dot{\varphi}$, therefore it is always accompanied by $\left.n_{t}^{(1)}=1\right)$.

Although it has a local form, the result is still different from the one in the standard scenario because non-Gaussianities are/can be enhanced by the particle content/creation concerning the perturbations depending on $k_{2,3}$ as we have discussed in section 6.1.

Cases $\left|k_{1} \eta_{c}^{\left(k_{s}\right)}\right| \gg 1$ and $\frac{k_{1}}{k_{S}} \frac{\Lambda}{H} \gg 1$

In this case, equations (71)-(72) and (766)-(77) yield, at leading order,

$$
\begin{aligned}
\delta_{\beta}\left\langle\zeta_{\vec{k}_{1}}(\eta) \zeta_{\vec{k}_{2}}(\eta) \zeta_{\vec{k}_{3}}(\eta)\right\rangle_{\lambda_{\mathrm{nms}}}=\epsilon\left|\beta_{k_{S}}\right| \frac{\lambda_{n m s}}{\sqrt{2 \epsilon}} \frac{\Lambda M_{\text {Planck }}}{H^{2}}\left(\frac{k_{S}}{k_{1}}\right)^{-1-\min (n, m, s)} \\
\left.\quad \times \sum_{j=2}^{3}\left\{\begin{array}{ll}
\left(\frac{H}{\Lambda} \frac{k_{S}}{k_{1}}\right)^{n+m+s} & \text { if } 1+\cos \left(\theta_{j}\right)>\frac{H}{\Lambda} \frac{1}{x_{1}} \\
{\left[\frac{H D}{\Lambda}\right)^{n+m+s}\left(1+c_{n_{t}^{(1)}}\left(\frac{k_{1} D}{k_{S}}\right)^{n_{t}^{(1)}-1}\right)} & \text { mis } \\
\left(\frac{H}{\Lambda} \frac{k_{S}}{k_{1}}\right)^{\frac{n+m+s}{\kappa+1}} & \text { mdr }
\end{array}\right] \quad \begin{array}{r}
\text { if } 1+\cos \left(\theta_{j}\right)<\frac{H}{\Lambda} \frac{1}{x_{1}}
\end{array}\right\} P_{\mathrm{st}}\left(k_{1}\right) P_{\mathrm{st}}\left(k_{S}\right) .
\end{aligned}
$$


Except for the case of folded configurations in modified initial state scenarios, it appears from (92) that higher-derivative couplings (larger values for $n, m, s$ ) would lead to a more pronounced growth for small $k_{1}$ since its exponent will be more negative. However, these contributions are very suppressed because of the factors $\left(\frac{H}{\Lambda}\right)^{n+m+s},\left(\frac{H}{\Lambda}\right)^{\frac{n+m+s}{\kappa+1}}$. The most interesting cases are thus those that are least suppressed. Considering the possible values for $n, m, s$, see section [5.3, this happens for instance for the minimal cubic coupling that we studied as a detailed example, where $n+m+s=1$ and $\min (n, m, s)=-1$, see (65), in which case (roughly, see (36)-(37), (46)-(47) for the details)

$\delta_{\beta}\left\langle\zeta_{\vec{k}_{1}}(\eta) \zeta_{\vec{k}_{2}}(\eta) \zeta_{\vec{k}_{3}}(\eta)\right\rangle_{\operatorname{min~cub}}=\epsilon\left|\beta_{k_{S}}\right| \sum_{j=2}^{3}\left\{\begin{array}{ll}\frac{k_{S}}{k_{1}} & \text { if } 1+\cos \left(\theta_{j}\right)>\frac{H}{\Lambda} \frac{1}{x_{1}} \\ {\left[\begin{array}{ll}H & \text { mis } \\ \left(\frac{\Lambda}{H}\right)^{\frac{\kappa}{\kappa+1}}\left(\frac{k_{S}}{k_{1}}\right)^{\frac{1}{\kappa+1}} & \text { mdr }\end{array}\right]} & \text { if } 1+\cos \left(\theta_{j}\right)<\frac{H}{\Lambda} \frac{1}{x_{1}}\end{array}\right\} P_{\mathrm{st}}\left(k_{1}\right) P_{\mathrm{st}}\left(k_{S}\right)$.

We see from (93) that in this case the form of the bispectrum in the squeezed limit has $i$ ) a nonlocal shape, with a scaling as $k_{1}^{-4}$ and with amplitude not too severely suppressed for non-folded configurations, ii) a non-local shape and enhanced amplitude (see also section 6.1) for (nearly) folded configurations in the case of modified dispersion relations, and iii) a local shape and greater enhancement for folded configurations in the modified initial state case. Interestingly, the scaling with $k_{1}$ of the contribution from (nearly) folded configurations in the case of modified dispersion relation captures the power $\kappa$ of the first momentum correction to the standard dispersion relation, see (92).

\subsection{Predictions for the different modified scenarios}

We have seen that, most interestingly, in modified scenarios the leading features of the bispectrum in the squeezed limit depend on very general aspects of the theory (exit from horizon, particle production, interference and accumulation with time), due to the modification of the Whightman functions in these scenarios. These aspects cannot determine the bispectrum in the squeezed limit in all details, but, even adopting a wide-range phenomenological approach, we have been able to determine both the leading dependence on the squeezed scale $k_{1}$ and the presence of enhancements of non-Gaussianities and their bounds.

We have seen that these features depend on the values (less or more than 1) of the quantities $\left|k_{1} \eta_{c}^{\left(k_{s}\right)}\right|$ and $\frac{k_{1}}{k_{S}} \frac{\Lambda}{H}$. Note that $\frac{k_{1}}{k_{S}}$ represents the sensitivity of the observation (the smallest ratio of scales that can be probed). We would like now to ask ourselves which kind of conditions or predictions the different theoretical scenarios impose or make about the values of $\left|k_{1} \eta_{c}^{\left(k_{s}\right)}\right|$ and $\frac{k_{1}}{k_{S}} \frac{\Lambda}{H}$, and, thus, what features of the bispectrum would be realized in the squeezed limit.

- BEFT modified initial state. In the Boundary Effective Field Theory approach, the initial condition for the fields is fixed at a time $\eta_{c}$ independently of the modes $k$ ("beginning of inflation"). Thus $\eta_{c}^{\left(k_{S}\right)}=\eta_{c}^{\left(k_{1}\right)}=\eta_{c}$ and since initial conditions are always fixed when modes were subhorizon, then $\left|k_{1} \eta_{c}^{\left(k_{1}\right)}\right| \gg 1 \Rightarrow\left|k_{1} \eta_{c}^{\left(k_{S}\right)}\right| \gg 1$.

Looking therefore at the analysis in sections 6.1, 6.2, we are in the cases of equations (85), (92), and the leading possible contribution is given by (93), see also (88). This means that if the BEFT is the correct physical approach to model the effects of very high energy physics from a low-energy point of view, then the largest possible contribution to the bispectrum for non-folded configurations would grow at large scales as $k_{1}^{-4}$, but with only moderately enhanced non-Gaussianities, whereas the contribution from folded configurations would be more enhanced, but growing only as $k_{1}^{-3}$.

- NPHS modified initial state. In the NPHS approach the boundary condition is mode-dependent, and imposed at the time given by $\left|k \eta_{c}^{(k)}\right|=\frac{\Lambda}{H} \gg 1$. The picture is that different modes are created at 
different times in a certain condition that fixes the initial state of the perturbations in the effective theory at scales lower than $\Lambda$.

When computing the three-point function, therefore, we cannot trace the interaction of the modes backward in time for a time earlier than the "creation time" for the largest of the considered modes: the prior evolution (and our ignorance about it) is encoded in the initial state. Thus, in the case of the squeezed limit, since $\eta_{c}^{\left(k_{S}\right)} \neq \eta_{c}^{\left(k_{1}\right)}$, it can happen that $\left|k_{1} \eta_{c}^{\left(k_{S}\right)}\right|=\frac{k_{1}}{k_{S}} \frac{\Lambda}{H}$ is larger or smaller than 1 (super- or sub-horizon condition) despite the fact that $\left|k_{1} \eta_{c}^{\left(k_{1}\right)}\right| \gg 1$, depending on the values of the scales at play in the specific model.

Therefore the bispectrum will behave in different ways for the various possibilities. Looking at the analysis in sections 6.1, 6.2, we see that the leading correction to the bispectrum would grow at large scales as $k_{1}^{-4}$ for non-folded configurations if the scale of new physics is such that $\Lambda>\frac{k_{S}}{k_{1}} H$, where $\frac{k_{1}}{k_{S}}$ is the smallest ratio of scales that can be probed, see (92), (93). On the other hand, for folded configurations, or when $\Lambda<\frac{k_{S}}{k_{1}} H$ (superhorizon condition), the contribution would be of the local type, growing at most as $k_{1}^{-3}$ as in the standard case, but will be enhanced, see (90), (91), (92), (93), (83).

An interesting outcome of this result is that since we have different signatures depending on whether $\frac{k_{1}}{k_{S}} \frac{\Lambda}{H}$ is larger or smaller than 1 , in case we could detect one or the other, say in the halo bias, and separate them from other kinds of non-Gaussianities (such as the secondary ones), we would obtain information on the magnitude of $\Lambda$ given our knowledge of the observation sensitivity $\frac{k_{1}}{k_{S}}$.

- Modified dispersion relations. In the scenarios with modified dispersion relations violating WKB at early times, $\frac{k_{1}}{k_{S}} \frac{\Lambda}{H}$ can be either smaller or greater than 1, depending on the value of the Lorentzbreaking scale $\Lambda$ and the sensitivity $\frac{k_{1}}{k_{S}}$ of the observation (smallest ratio that can be probed). In the case when $\frac{k_{1}}{k_{S}} \frac{\Lambda}{H}>1$, there would be contributions to the bispectrum growing as fast as $k_{1}^{-3-N}$ with $N$ only bound by the specific model, and in principle also possibly large, due to the presence of higher-derivative Lorentz-breaking couplings, see (92). However, we have found that those contributions are severely suppressed by powers $\left(\frac{H}{\Lambda}\right)^{M}, M \geq N$.

The least-suppressed possible contributions (which could actually be enhanced) show at leading order a growth as $k_{1}^{-4}$ for non-folded configurations, and as slower powers $k_{1}^{-r}$, with $r$ fractional, for the (nearly) folded ones, see (92), (93). Remarkably, in the latter case, the exponent of $k_{1}$ is determined by the momentum power of the lowest high-energy correction to the standard dispersion relation, see (93), (45), and it would thus be of great observational interest.

On the other hand, in the case $\Lambda<\frac{k_{S}}{k_{1}} H$, the leading contribution would be of the local type growing at most as $k_{1}^{-3}$, see (91), but it could be enhanced, see (83).

Once again, if we could observe one or the other behaviour of the bispectrum, we could infer whether $\frac{k_{1}}{k_{S}} \frac{\Lambda}{H}$ is either larger or smaller than 1 , and thus obtain information on the magnitude of the Lorentz-breaking scale from our knowledge of the probed $k_{1}$.

\section{Comments on the halo bias}

We will briefly comment here on the implications of our results for the halo bias, leaving a more detailed analysis for future research. The halo bias depends on the bispectrum and the spectrum 


$$
\begin{aligned}
\frac{\Delta b_{h}\left(k_{1}, R\right)}{b_{h}}=\frac{\delta_{c}}{D(z) \mathcal{M}_{R}\left(k_{1}\right)} & \frac{1}{8 \pi^{2} \sigma_{R}^{2}} \int_{0}^{\infty} d k_{2} k_{2}^{2} \mathcal{M}_{R}\left(k_{2}\right) \\
& \times \int_{-1}^{1} d \xi \mathcal{M}_{R}\left(\sqrt{k_{1}^{2}+k_{2}^{2}+2 k_{1} k_{2} \xi}\right) \frac{B\left(k_{2}, \sqrt{k_{1}^{2}+k_{2}^{2}+2 k_{1} k_{2} \xi}, k_{1}\right)}{P\left(k_{1}\right)}
\end{aligned}
$$

where $\sigma_{R}^{2}$ is the variance of the dark matter density perturbations smoothed on a scale of Lagrangian radius $R, \delta_{c}$ is the critical threshold for the collapse of a spherical object (for a matter-dominated universe $\left.\delta_{c}=1.686\right), D(z)$ is the linear growth factor normalized to be $D(z)=(1+z)^{-1}$ during matter domination, $P$ and $B$ are the two-point correlator 27 and bispectrum for $\zeta$, see equations (2)(3), and $\mathcal{M}_{R}$ is the linear relation between the dark matter density perturbations smoothed on a scale $R$ and the primordial curvature perturbation:

$$
\mathcal{M}_{R}(k) \equiv \frac{2 k^{2}}{5 \Omega_{m} H_{0}^{2}} T(k) W_{R}(k)
$$

where $\Omega_{m}$ is the present time fractional density of matter. Finally, $H_{0}$ is the present Hubble rate, $T$ is the transfer function normalized to one on large scales and $W_{R}$ is the filter function with characteristic scale $R$.

The halo bias depends on both the primordial bispectrum and the subsequent gravitational processing which generates additional non-Gaussianities. These General Relativity corrections have been studied for example in [13] and it has been shown that they give a typical contribution to the halo bias, which could permit to distinguish them. We will therefore comment only the primordial contributions.

The behaviour of the halo bias at large scales has been computed for the local, equilateral and folded templates for the primordial non-Gaussianities in [13] (see also [6 9, 14]), finding that the bias goes approximately as $k_{1}^{-2}$ with the local bispectrum template, as a constant in $k_{1}$ with the equilateral, and as $k_{1}^{-1}$ with the folded.

The latter result is the one that can be compared with those we have found for the scenario of the modified initial state scenario, since that template was indeed proposed as an approximation to model the bispectrum in that case [15]. In fact, we have shown that it leads to the wrong result for the squeezed limit: the folded bispectrum template goes as $k_{1}^{-2}$, which is very different from the true bispectrum behaviour found in sections 5 and 6 . The reason for this is that the template does not depend on the additional scale(s) $\eta_{c}, \Lambda$ and thus its squeezed limit is different than the one that actually occurs in the rigorous field theory computation.

We have indeed found in sections 5 and 6 that in the squeezed limit the leading contribution to the bispectrum grows as $k_{1}^{-4}$ or $k_{1}^{-3}$, depending on the compared magnitudes of the scale of new physics and the sensitivity of the observation (the smallest observable squeezed momentum scale). Therefore, from (94) and the results in sections 5 and 6 , the halo bias for modified initial states would approximately grow either as $k_{1}^{-3}$ or $k_{1}^{-2}$ for small $k_{1}$, and not as $k_{1}^{-1}$ as predicted by the template.

Similarly, in the scenario of modified dispersion relations violating WKB at early times, equation (94) and the results in sections 5 and 6, would also lead to a bias that approximately grows either as $k_{1}^{-3}$ or $k_{1}^{-2}$ for small $k_{1}$ for non-folded configurations, again depending on the relative magnitudes of the high-energy scales and the sensitivity of the observations. (Nearly) folded configurations would

\footnotetext{
${ }^{26}$ The formula (94), obtained in [13, is calculated in a local-Lagrangian-biasing scheme. Reference 14 has a similar but not identical formula calculated using a point-break splitting method. As discussed in 14, the formulas agree for large scales, but differ for intermediate scale, where the point-break splitting method involves more important approximations. It seems however that the formula (94) is the one that is better in agreement with the simulations [8], see also [12.

${ }^{27}$ In the literature on the halo bias, the two-point correlator is often called the spectrum [13, 14, not to be confused with the quantity $\mathcal{P}=\frac{k^{3}}{2 \pi^{2}} P$, also called spectrum, usual in the CMBR literature.
} 
lead to different, subleading scalings with $k_{1}$, determined by the exponent of the first correction to the standard dispersion relation for small momenta.

As for the magnitude of the halo bias, a feature relevant for the observations of the bias is that, as we have seen, the high-energy modifications could enhance the single-field non-Gaussianities in the squeezed limit, compared to the practically undetectable level that is present in the standard scenarios of single-field models with Bunch-Davies vacuum and standard kinetic terms. However, the final magnitude of the non-Gaussianities depends also on the specific model, which determines the precise form and magnitude of the coefficients $\beta_{k_{S}}$ entering the equations.

\section{Final discussion and conclusion}

In this article we have analysed the squeezed limit of the bispectrum in single-fields models of inflation when modifications of the theory at very high energy are present. Due to the large number of inflationary models available, it is useful to look at general features that can be obtained when these modifications are rather generic and simple.

In particular, we have considered 1) the presence of terms with higher derivatives, which are not suppressed any more beyond a certain momentum scale and modify the dispersion relations of the fields, and 2) the possibility of initial condition for the solution of the field equation that parametrize in a simple and generic way our ignorance on the physics at very high energies. In the case 1) the most significant results occur for dispersion relation that violate WKB (for a short time) at early times; in the case 2) we have considered both NPHS and BEFT approaches, where the initial condition is imposed respectively in a scale-invariant and non-scale-invariant way.

The leading contribution to the squeezed limit appears dominated by very general features of the scenarios (particle content/creation at early times, interference and cumulation with time) and thus it is possible to obtain general results and bounds. From the more mathematical point of view, the reason for the leading differences with the standard result is the modifications of the Whightman function of the theory.

The result and bounds that we have found are interesting in two respects: on the one hand, they show that non-Gaussianities could be enhanced by high energy modifications of the theory, which would improve the possibility of detection of those phenomena. On the other, the behaviour of the bispectrum at large scale in these scenarios is distinctive, and differs from the one that has been found in the standard scenarios, with Bunch-Davies vacuum and standard dispersion relation. It is also different from that found in previous works using the approximate template proposed in [15] for the modified initial state approach.

Our results show that it could be possible to obtain various different pieces of information about the features of the high energy physics theory from large scale observations such as those of the halo bias, if the signatures we have found were detected. In fact, the enhancements and the dependence of the bispectrum on the small wavenumber $k_{1}$ in the realistic (observable) squeezed limit change in relation with the magnitude of the scale of new physics. Hence, if able to detect certain behaviours of the bispectrum, one can place bounds on the new scale of physics.

Of course, other sources could give origin to sizable non-Gaussianities, for example in multi-field models. An interesting question for future research would then be if the effects due to high-energy modifications of the theory that we have considered can be distinguished from those. For example, the dependence on the small mode $k_{1}$ of the halo bias could be different, or the enhancement factors could be absent or of different magnitude. We leave this as an interesting outlook for future research. 


\section{Acknowledgments}

The author is supported by a Postdoctoral F.R.S.-F.N.R.S. research fellowship via the Ulysses Incentive Grant for the Mobility in Science and is "chercheur de recherche" F.R.S.-F.N.R.S..

\section{A Appendices}

\section{A.1 Solution of the field equation in the case of modified dispersion relations}

We parametrize the modifications to the dispersion relation via a generic function $F\left(\frac{H}{\Lambda} k \eta\right)$ :

$$
\omega(\eta, k)=a(\eta) \omega_{\text {phys }}(p)=a(\eta) p F\left(-\frac{p}{\Lambda}\right)=k F\left(\frac{H}{\Lambda} k \eta\right), \quad F(x \rightarrow 0) \rightarrow 1, \quad \frac{H}{\Lambda} \ll 1,
$$

with the only request that the WKB conditions be violated for a short period of time at early times. The generic shape of such a dispersion relation is in figure 1. The approximate solution of the equation of motion (6) in these cases is given in equation (11), where, see [20]:

- in regions I and III, in terms of the convenient variable $\frac{H}{\Lambda} k \eta \equiv y_{k}$ :

$$
u_{1}\left(y_{k}\right)=\frac{e^{-i \frac{\Lambda}{H} \Omega_{\mathrm{F}}\left(y_{k}\right)}}{\sqrt{2 k U\left(y_{k}\right)}}, \quad u_{2}\left(y_{k}\right)=u_{1}^{*}\left(y_{k}\right)=\frac{e^{+i \frac{\Lambda}{H} \Omega_{\mathrm{F}}\left(y_{k}\right)}}{\sqrt{2 k U\left(y_{k}\right)}}
$$

with

$$
\Omega_{\mathrm{F}}\left(y_{k}\right)=\int^{y_{k}} U\left(y_{k}^{\prime}\right) d y_{k}^{\prime}, \quad U\left(y_{k}\right) \equiv F+\epsilon^{2}\left(-\frac{\partial_{y}^{2} F}{4 F^{2}}+3 \frac{\left(\partial_{y} F\right)^{2}}{8 F^{3}}-\frac{1}{F y_{k}^{2}}\right),
$$

- in region IV, where $\omega(\eta, k) \sim k$ and $k \eta \lesssim 1$,

$$
\mathcal{V}_{1,2}=\left.\sqrt{-\eta} H_{\nu}^{(1,2)}(-k \eta)\right|_{k \eta \lesssim 1}, \quad \nu \sim \frac{3}{2}
$$

- in region II, the partial solution $\mathcal{U}_{1,2}(\eta, k)$ can be found either by solving in details the specific equation, if one has a favourite model, or, more generally, by expanding the frequency around the minimum $\omega_{0}$ at $\eta=\eta_{\min }$ as

$$
\omega^{2}=a^{2} \omega_{\text {phys }}^{2} \simeq \frac{1}{\eta^{2} H^{2}}\left(\omega_{0}^{2}+\omega_{0} \omega_{\text {phys }}^{\prime \prime}\left(\eta_{\min }\right)\left(\eta-\eta_{\min }\right)^{2}\right)
$$

so that

$$
\begin{gathered}
f_{k}(\eta)_{\mathrm{II}}=\sum_{i=1}^{2} B_{i} \mathcal{U}_{i}(\eta, k)=B_{1} W\left(i \kappa \eta_{\min }, \sigma, 2 i \kappa \eta\right)+B_{2} W\left(-i \kappa \eta_{\min }, \sigma,-2 i \kappa \eta\right) \\
\kappa \equiv \frac{\sqrt{\omega_{0} \omega_{\text {phys }}^{\prime \prime}\left(\eta_{\min }\right)}}{H} \quad \sigma \equiv \frac{\sqrt{9 H^{2}-4 \omega_{0}^{2}-4 H^{2} \kappa^{2} \eta_{\min }^{2}}}{2 H}
\end{gathered}
$$

where $W(a, b, z)$ is the Whittacker function. As explained in [20], this approximation is welljustified also because backreaction constraints the interval $\left[\eta_{\mathrm{I}}, \eta_{\mathrm{II}}\right]$ to be very small $(\Delta<1)$, so that $\eta \sim \eta_{\min }$ for $\eta, \eta_{\min } \in\left[\eta_{\mathrm{I}}, \eta_{\mathrm{I}}\right]$. In any case, it actually turns out that the details of the solution in region II are not important for what concerns the leading contribution to the bispectrum [20]. 
By asking for the continuity of the function and its first derivative, and imposing the Wronskian condition $\mathcal{W}\left\{f, f^{*}\right\}=-i$ to have the standard commutation relations in the quantum theory, we obtain in full generality

$$
\begin{aligned}
D_{1} & =\frac{\sqrt{\pi}}{2} e^{i \frac{\pi}{2} \nu+i \frac{\pi}{4}} \alpha_{k} & D_{2} & =\frac{\sqrt{\pi}}{2} e^{-i \frac{\pi}{2} \nu-i \frac{\pi}{4}} \beta_{k} \\
B_{1} & =\left.\frac{\mathcal{W}\left\{\varsigma_{k} u_{1}, \mathcal{U}_{2}\right\}}{\mathcal{W}\left\{\mathcal{U}_{1}, \mathcal{U}_{2}\right\}}\right|_{\eta_{\mathrm{I}}} & B_{2} & =-\left.\frac{\mathcal{W}\left\{\varsigma_{k} u_{1}, \mathcal{U}_{1}\right\}}{\mathcal{W}\left\{\mathcal{U}_{1}, \mathcal{U}_{2}\right\}}\right|_{\eta_{\mathrm{I}}} \\
\alpha_{k} & =\left.\frac{\mathcal{W}\left\{B_{1} \mathcal{U}_{1}+B_{2} \mathcal{U}_{2}, u_{2}\right\}}{\mathcal{W}\left\{u_{1}, u_{2}\right\}}\right|_{\eta_{\mathrm{II}}} & \beta_{k} & =-\left.\frac{\mathcal{W}\left\{B_{1} \mathcal{U}_{1}+B_{2} \mathcal{U}_{2}, u_{1}\right\}}{\mathcal{W}\left\{u_{1}, u_{2}\right\}}\right|_{\eta_{\mathrm{II}}},
\end{aligned}
$$

where $\mathcal{W}$ is the Wronskian. We choose $\varsigma_{k}=1$ picking up the usual adiabatic vacuum. The Wronskian condition also imposes $\left|\alpha_{k}\right|^{2}-\left|\beta_{k}\right|^{2}=1$. By expanding for small $\Delta$ around $\eta_{\mathrm{I}}$, we obtain equation (13) in the text.

Finally, the parameter signalling the WKB violation is [20]

$$
\mathcal{Q}=-\left(-\frac{\omega^{\prime \prime}}{4 \omega^{2}}+3 \frac{\omega^{\prime 2}}{8 \omega^{3}}-\frac{1}{\omega \eta^{2}}\right)^{2}+\frac{\omega^{\prime \prime}}{2 \omega}-3 \frac{\omega^{\prime 2}}{4 \omega^{2}}-\frac{U^{\prime \prime}}{2 U}+3 \frac{U^{\prime 2}}{4 U^{2}}
$$

\section{A.2 Formulas for Whightman functions and their time derivatives}

The bispectrum is computed at a time $\eta$ when all momenta have exit the horizon, therefore the Whightman functions have the general form

$$
G_{k}\left(\eta \sim 0, \eta^{\prime}\right)=\lim _{\eta \rightarrow 0} \frac{H^{2}}{\dot{\phi}^{2}} \frac{f_{k}(\eta)}{a(\eta)} \frac{f_{k}^{*}\left(\eta^{\prime}\right)}{a\left(\eta^{\prime}\right)}
$$

We list here the explicit formulas obtained by substituting in the above equations the field modes functions $f_{k}$ for $\zeta$ in the various scenarios we consider. Due to the presence of positive- and negativeenergy solutions, the Whightman function will also have positive- and negative-energy parts, which we indicate with $G_{k}^{ \pm}$.

- Standard scenario and modified initial state. From equations (8), (15)

$$
G_{k}^{ \pm}\left(0, \eta^{\prime}\right)= \pm \frac{H^{2}}{\dot{\phi}^{2}} \frac{H^{2}}{2 k^{3}}\left(1 \mp i k \eta^{\prime}\right) e^{ \pm i k \eta^{\prime}}, \quad \partial_{\eta^{\prime}} G_{k}^{ \pm}\left(0, \eta^{\prime}\right)= \pm \frac{H^{2}}{\dot{\phi}^{2}} \frac{H^{2}}{2 k^{3}} k^{2} \eta^{\prime} e^{ \pm i k \eta^{\prime}}
$$

- Modified dispersion relations. From equation (11), we find that in region IV the Whightman function is the same as the one in equation (107), while in region III it is obtained from (97). Thus,

$$
G_{k}^{ \pm}\left(\eta \sim 0, \eta^{\prime}\right)=\frac{H^{2}}{\dot{\phi}^{2}} \frac{e^{i \frac{\pi}{2}}}{2 k^{2}} \frac{H}{a\left(\eta^{\prime}\right)} \chi^{(*)}\left(k, \eta^{\prime}\right) e^{ \pm i \frac{\Lambda}{H} \Omega\left(k, \eta^{\prime}\right)}, \quad \chi\left(k, \eta^{\prime}\right)= \begin{cases}\frac{1}{\sqrt{U\left(k, \eta^{\prime}\right)}} & \text { in region III } \\ \left(1-\frac{i}{k \eta^{\prime}}\right) & \text { in region IV }\end{cases}
$$

where $\Omega\left(k, \eta^{\prime}\right)$ is equal to $\Omega_{\mathrm{F}}\left(k, \eta^{\prime}\right)$ in region III and to $k \eta^{\prime}$ in region IV. The derivative of the Whightman function is

$$
\partial_{\eta^{\prime}} G_{k}^{ \pm}\left(\eta \sim 0, \eta^{\prime}\right)=-\frac{H^{2}}{\dot{\phi}^{2}} \frac{e^{i \frac{\pi}{2}} H}{2 k} \frac{\gamma^{(*)}\left(k, \eta^{\prime}\right)}{a\left(\eta^{\prime}\right)} e^{ \pm i \frac{\Lambda}{H} \Omega\left(k, \eta^{\prime}\right)}, \quad \gamma\left(k, \eta^{\prime}\right)= \begin{cases}\frac{1}{\sqrt{U}}\left(\frac{1}{2 k} \frac{U^{\prime}}{U}+i U+\frac{\mathcal{H}}{k}\right) & \text { in region III } \\ i & \text { in region IV }\end{cases}
$$

where the suffix ${ }^{(*)}$ means that the positive-energy solution is associated with $\gamma^{*}, \chi^{*}$, while the negative-energy with $\gamma, \chi$. Finally, $\mathcal{H}$ is the conformal Hubble scale. 


\section{A.3 Time integral in bispectrum computations with modified dispersion relations}

When computing the bispectrum, we have encountered various Laplace integrals of the form

$$
\mathcal{J}_{j}^{(w)}=\int_{y_{\text {II }}}^{y} d y^{\prime} y^{\prime w-1} e^{i \frac{\Lambda}{H} x_{1} \tilde{v}_{\theta_{j}}\left(y^{\prime}\right)} h\left(x_{1}, x_{S}, \theta_{j}, y^{\prime}\right),
$$

see equations (39), (158), (75), where $w$ and $h\left(x_{1}, x_{S}, \theta_{j}, y^{\prime}\right)$ change in the different integrals and the variables are defined in (38).

In particular, in the case of the minimal coupling interaction (18), the integral present in the correction of order $\beta_{k}$ to the bispectrum, see equation (39), has the form of (110) with $w=1$ and $h\left(\{x\}, \theta_{j}, y^{\prime}\right) \rightarrow g\left(\{x\}, \theta_{j}, y^{\prime}\right)$, where [20]

$$
g\left(\left\{x_{h \neq j}\right\}, x_{j}, y^{\prime}, m\right) \equiv \prod_{h \neq j} \gamma^{*}\left(x_{h}, y^{\prime}\right) \tilde{\gamma}\left(x_{j}, y^{\prime}\right) e^{i \frac{H}{\Lambda} \int^{y^{\prime}}\left(\sum_{h \neq j} S_{2}\left(x_{h}\right)+(-1)^{m} S_{2}\left(x_{j}\right)\right)}
$$

with $\gamma$ defined in (109) and

$$
S_{2}\left(x_{i}, y\right)=-\frac{\partial_{y}^{2} \omega\left(x_{i} y\right)}{4 \omega\left(x_{i} y\right)^{2}}+3 \frac{\left(\partial_{y} \omega\left(x_{i} y\right)\right)^{2}}{8 \omega\left(x_{i} y\right)^{3}}-\frac{1}{\omega\left(x_{i} y\right) y^{2}}, \quad \tilde{\gamma}\left(x, y^{\prime}\right)=\left\{\begin{array}{ll}
\gamma^{*}\left(x, y^{\prime}\right) & \text { if } m=0 \\
\gamma\left(x, y^{\prime}\right) & \text { if } m=1
\end{array} .\right.
$$

In the squeezed limit, for $m=1$ and $j=2,3$, looking at (98) and (109), we obtain

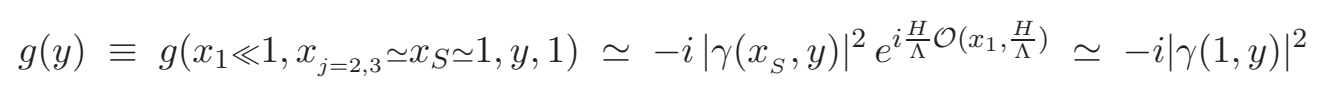

In particular, the $\mathcal{O}(1)$ coefficient appearing in the result for the contribution to the bispectrum for $\frac{\Lambda}{H} x_{1} \ll 1$ in the last line of (43) and of (47), is a purely numerical factor, which reads in details:

$$
\int_{-1}^{0} d y^{\prime}\left|\gamma\left(1, y^{\prime}\right)\right|^{2} \sim \mathcal{O}(1)
$$

In the case, instead, of the higher derivative interaction in equation (48), the integral appearing in equation (58) has the form of (110) with $w=3$ and $h\left(\{x\}, \theta_{j}, y^{\prime}\right) \rightarrow q\left(\{x\}, \theta_{j}, y^{\prime}\right)$, where [20]

$$
\begin{aligned}
q\left(\left\{x_{h}\right\}, x_{j}, y\right)=\left(6 \prod_{h \neq j} \gamma^{*}\left(x_{h}\right) \gamma\left(x_{j}\right)\right. & +\frac{2 \vec{x}_{j+1} \cdot \vec{x}_{j+2}}{x_{j+1} x_{j+2}} \chi^{*}\left(x_{j+1}\right) \chi^{*}\left(x_{j+2}\right) \gamma\left(x_{j}\right)+\frac{2 \vec{x}_{j+1} \cdot \vec{x}_{j}}{x_{j+1} x_{j}} \chi^{*}\left(x_{j+1}\right) \chi\left(x_{j}\right) \gamma^{*}\left(x_{j+2}\right) \\
& \left.+\frac{2 \vec{x}_{j+2} \cdot \vec{x}_{j}}{x_{j+2} x_{j}} \chi^{*}\left(x_{j+2}\right) \chi\left(x_{j}\right) \gamma^{*}\left(x_{j+1}\right)\right) e^{i \frac{H}{\Lambda} \int^{y}\left(\sum_{h \neq j} S_{2}\left(x_{h}\right)-S_{2}\left(x_{j}\right)\right)}, \quad(115)
\end{aligned}
$$

and $S_{2}$ has been defined in equation (112) while $\chi$ in (108). We write explicitly only the dependence on $x$ for $\gamma, \chi$ to avoid cluttering the formula. The labels of the $x$ 's are defined modulo 3 , here.

In the squeezed limit, for $j=2,3$, looking at (98) and (109), the integral of the leading contribution has

$$
q\left(\theta_{j}, x_{1}, y\right) \equiv q\left(x_{1} \ll 1, x_{j=2,3} \simeq x_{S} \simeq 1, y\right) \simeq-6 i\left|\gamma^{*}(1, y)\right|^{2}+2 i|\chi(1, y)|^{2}-4 i \chi^{*}\left(x_{1}, y\right) \cos \left(\theta_{j}\right),
$$

so that, again, the $\mathcal{O}(1)$ coefficient appearing in the result for the contribution to the bispectrum for $\frac{\Lambda}{H} x_{1} \ll 1$, see last line of (46), is a purely numerical factor

$$
\int_{-1}^{0} d y^{\prime}\left(3\left|\gamma\left(1, y^{\prime}\right)\right|^{2}-\left|\chi\left(1, y^{\prime}\right)\right|^{2}\right) \sim \mathcal{O}(1)
$$




\begin{tabular}{||c|c|c|c||}
\hline & $|y| \ll 1$ & $y=y_{\mathrm{II}}$ & $y \simeq y_{*}$ \\
\hline \hline$F\left(x_{S}, y\right)$ & $1+(-y)^{\kappa} F^{(k)}$ & $F(-1)$ & $F_{*}+F_{*}^{\left(\nu_{*}\right)}\left|y-y_{*}\right|^{\nu_{*}}$ \\
\hline$\tilde{v}_{\theta_{j}}(y)$ & $y v_{\theta_{j}}-(-y)^{\kappa+1} F^{(k)} \cos \left(\theta_{j}\right)$ & $-1-F(-1) \cos \left(\theta_{j}\right)$ & $\begin{array}{c}\tilde{v}_{\theta_{j}}\left(y_{*}\right)+\tilde{v}_{\theta_{j}}^{\left(\nu_{*}\right)}\left(y_{*}\right)\left|y-y_{*}\right|^{\nu_{*}} \\
\text { or }\end{array}$ \\
\hline$g(y)$ & $-i\left(1+|y|^{\kappa} F^{(k)}\right)$ & & $\begin{array}{c}\tilde{v}_{\theta_{j}}\left(y_{*}\right)+\tilde{v}_{\theta_{j}}^{\left(\nu_{*}\right)}\left(y_{*}\right) \\
\times \mid y-y_{*} \nu^{\nu_{*}} \operatorname{sign}\left(y-y_{*}\right)\end{array}$ \\
\hline$q\left(\theta_{j}, x_{1}, y\right)$ & $-4 i\left(v_{\theta_{j}}+2|y|^{\kappa} F^{(k)}\right)$ & $-6 i\left|\gamma^{*}(1,-1)\right|^{2}+2 i U(1,-1)^{-1}$ & $\left(y-y_{*}\right)^{\mu_{*}-1} g^{\left(\mu_{*}-1\right)}\left(y_{*}\right)$ \\
\hline
\end{tabular}

Table 1: Behaviour of functions entering the integrands in equations (39), (58), (75) close to the boundary points (limits of integration) $y_{\mathrm{II}} \sim-1$ and $y \sim-\frac{H}{\Lambda} \ll-1$, and close to the possible stationary point(s) $y_{*}$ in the squeezed limit $x_{1} \ll 1, x_{S} \simeq 1$.

Similarly, we find that the $\mathcal{O}(1)$ coefficients appearing in the result for the contribution (75) to the bispectrum for $\frac{\Lambda}{H} x_{1} \ll 1$, see last line of (77), are given by

$$
\left.\int_{-1}^{0} d y^{\prime}\left(y^{\prime}\right)^{u} \gamma^{*}\left(1, y^{\prime}\right)^{n_{t}^{(h)}} \gamma^{*}\left(1, y^{\prime}\right)^{n_{t}^{(h+1)}}\right|_{h \neq j} \sim \mathcal{O}(1)
$$

where $n_{t}^{(h)}$ has been defined below (75) and $u$ is equal to $v / v_{t}$, defined above (175), in the first/second coefficient.

For $\frac{\Lambda}{H} x_{1} \gg 1$, the integrals like (110) are dominated by the contribution of boundary and stationary points, where the oscillations due to the large phase of the integrand are reduced. It is therefore important to study the behavior at leading order of the integrands in (39), (58), (75) close to the boundary points (limits of integration) $y_{\text {II }} \sim-1$ and $y \sim-\frac{H}{\Lambda} \ll-1$ and to the possible stationary point(s) $\left\{y_{*}\right\}$. In table 1, we report the leading and next-to-leading behaviour.

In the table, we include also the function $F\left(x, y^{\prime}\right)$, defined in (96), and now written in terms of the variables (38). From its definition (96), it appears that its expansion in powers of $\frac{p}{\Lambda}=-y$ for $|y| \ll 1$ is in fact the expansion for the effective frequency from the effective action. In particular, $\kappa+1$ is thus the power of the first correction to the standard physical dispersion relation (model dependent):

$$
\omega_{\text {phys }}(p) \sim p\left(1+\left(\frac{p}{\Lambda}\right)^{\kappa} F^{(\kappa)}+\cdots\right) .
$$

In expanding $g(y), q\left(\theta_{j}, x_{1}, y\right)$ - see (113), (116) - we have taken into account the WKB conditions. The powers $\nu_{*}, \mu_{*}, \tau_{*}$ are model dependent. We wish to be generic, so $\kappa, \nu_{*}, \mu_{*}, \tau_{*}$ can be fractional or integer 28 . Then, since $F=\frac{\omega_{\text {phys }}}{p}$ is a real function, its expansion must be clearly in powers of $|y|,\left|y-y_{*}\right|$, with real coefficients, for generic $\nu_{*}, \kappa$. Similarly, it must be so for $\tilde{v}_{\theta_{j}}(x, y) \Lambda=-\left(p+\omega_{\text {phys }}(p)\right)$, which is a real function as well (in table 1 we have been very generic concerning its expansion around $y_{*}$ ).

\section{References}

[1] E. Komatsu et al., arXiv:0902.4759 [astro-ph.CO]

\footnotetext{
${ }^{28}$ Given the WKB conditions, it is $\mu_{*} \geq 1$, while $\tau_{*} \geq 0$.
} 
[2] V. Desjacques and U. Seljak, Class. Quant. Grav. 27 (2010) 124011 arXiv:1003.5020 [astroph.CO]].

[3] N. Dalal, O. Dore, D. Huterer and A. Shirokov, Phys. Rev. D 77, 123514 (2008) arXiv:0710.4560 [astro-ph]].

[4] S. Matarrese and L. Verde, Astrophys. J. 677 (2008) L77 [arXiv:0801.4826 [astro-ph]].

[5] A. Slosar, C. Hirata, U. Seljak, S. Ho and N. Padmanabhan, JCAP 0808, 031 (2008) arXiv:0805.3580 [astro-ph]].

[6] S. Shandera, N. Dalal and D. Huterer, JCAP 1103 (2011) 017 arXiv:1010.3722 [astro-ph.CO]].

[7] V. Desjacques and U. Seljak, Phys. Rev. D 81 (2010) 023006 [arXiv:0907.2257 [astro-ph.CO]].

[8] C. Wagner, L. Verde and L. Boubekeur, JCAP 1010 (2010) 022 [arXiv:1006.5793 [astro-ph.CO]].

[9] K. M. Smith and M. LoVerde, JCAP 1111 (2011) 009 [arXiv:1010.0055 [astro-ph.CO]].

[10] J. M. Maldacena, JHEP 0305, 013 (2003) arXiv:astro-ph/0210603.

[11] P. Creminelli and M. Zaldarriaga, JCAP 0410 (2004) 006 arXiv:astro-ph/0407059.

[12] P. Creminelli, G. D’Amico, M. Musso and J. Norena, arXiv:1106.1462 [astro-ph.CO].

[13] L. Verde and S. Matarrese, Astrophys. J. 706 (2009) L91 [arXiv:0909.3224 [astro-ph.CO]].

[14] F. Schmidt and M. Kamionkowski, Phys. Rev. D 82 (2010) 103002 arXiv:1008.0638 [astroph.CO]].

[15] P. D. Meerburg, J. P. van der Schaar and P. S. Corasaniti, JCAP 0905, 018 (2009) arXiv:0901.4044 [hep-th]].

[16] J. Martin and R. H. Brandenberger, Phys. Rev. D 63, 123501 (2001) arXiv:hep-th/0005209.

[17] J. Martin and R. H. Brandenberger, Phys. Rev. D 65, 103514 (2002) arXiv:hep-th/0201189.

[18] J. Martin and R. Brandenberger, Phys. Rev. D 68 (2003) 063513 arXiv:hep-th/0305161.

[19] M. Lemoine, M. Lubo, J. Martin and J. P. Uzan, Phys. Rev. D 65 (2002) 023510 arXiv:hep-th/0109128.

[20] D. Chialva, JCAP 1201, 037 (2012) [arXiv:1106.0040 [hep-th]].

[21] D. Seery and J. E. Lidsey, JCAP 0506 (2005) 003 arXiv:astro-ph/0503692.

[22] X. Chen, M. x. Huang, S. Kachru and G. Shiu, JCAP 0701 (2007) 002 arXiv:hep-th/0605045.

[23] S. Renaux-Petel, JCAP 1010 (2010) 020 [arXiv:1008.0260 [astro-ph.CO]].

[24] C. Burrage, R. H. Ribeiro and D. Seery, JCAP 1107 (2011) 032 [arXiv:1103.4126 [astro-ph.CO]].

[25] J. Khoury and F. Piazza, JCAP 0907 (2009) 026 [arXiv:0811.3633 [hep-th]].

[26] J. Khoury and P. J. Steinhardt, Phys. Rev. Lett. 104 (2010) 091301 arXiv:0910.2230 [hep-th]].

[27] J. Khoury and P. J. Steinhardt, Phys. Rev. D 83 (2011) 123502 [arXiv:1101.3548 [hep-th]]. 
[28] D. Baumann, L. Senatore and M. Zaldarriaga, JCAP 1105 (2011) 004 [arXiv:1101.3320 [hep-th]].

[29] C. Wagner and L. Verde, arXiv:1102.3229 [astro-ph.CO].

[30] I. Agullo, L. Parker, Phys. Rev. D83 (2011) 063526. arXiv:1010.5766 [astro-ph.CO]].

[31] J. Ganc, Phys. Rev. D 84 (2011) 063514 [arXiv:1104.0244 [astro-ph.CO]].

[32] U. H. Danielsson, Phys. Rev. D 66, 023511 (2002) arXiv:hep-th/0203198.

[33] U. H. Danielsson, JHEP 0207 (2002) 040 arXiv:hep-th/0205227.

[34] R. Easther, B. R. Greene, W. H. Kinney and G. Shiu, Phys. Rev. D 66 (2002) 023518 hep-th/0204129.

[35] K. Schalm, G. Shiu and J. P. van der Schaar, JHEP 0404 (2004) 076 arXiv:hep-th/0401164.

[36] K. Schalm, G. Shiu and J. P. van der Schaar, AIP Conf. Proc. 743 (2005) 362 arXiv:hep-th/0412288.

[37] F. Nitti, M. Porrati and J. -W. Rombouts, Phys. Rev. D 72 (2005) 063503 hep-th/0503247.

[38] R. Holman and A. J. Tolley, JCAP 0805, 001 (2008) arXiv:0710.1302 [hep-th]].

[39] S. Weinberg, Phys. Rev. D 77 (2008) 123541 [arXiv:0804.4291 [hep-th]].

[40] U. H. Danielsson, JHEP 0212 (2002) 025 [arXiv:hep-th/0210058].

[41] M. B. Einhorn and F. Larsen, Phys. Rev. D 68 (2003) 064002 arXiv:hep-th/0305056.

[42] A. Vilenkin and L. H. Ford, Phys. Rev. D 26 (1982) 1231.

[43] A. Dey and S. Paban, JCAP 1204 (2012) 039 [arXiv:1106.5840 [hep-th]].

[44] G. Shiu and J. Xu, Phys. Rev. D 84 (2011) 103509 [arXiv:1108.0981 [hep-th]].

[45] M. G. Jackson and K. Schalm, Phys. Rev. Lett. 108 (2012) 111301 [arXiv:1007.0185 [hep-th]].

[46] P. Horava, Phys. Rev. D79, 084008 (2009). arXiv:0901.3775 [hep-th]].

[47] D. J. H. Chung and K. Freese, Phys. Rev. D 61 (2000) 023511 arXiv:hep-ph/9906542.

[48] D. J. H. Chung and K. Freese, Phys. Rev. D 62, 063513 (2000) arXiv:hep-ph/9910235.

[49] D. J. H. Chung, E. W. Kolb and A. Riotto, Phys. Rev. D 65, 083516 (2002) arXiv:hep-ph/0008126.

[50] C. Csaki, J. Erlich and C. Grojean, Nucl. Phys. B 604, 312 (2001) arXiv:hep-th/0012143.

[51] S. L. Dubovsky, JHEP 0201, 012 (2002) arXiv:hep-th/0103205.

[52] D. Mattingly, Living Rev. Rel. 8 (2005) 5 arXiv:gr-qc/0502097.

[53] T. Jacobson, S. Liberati and D. Mattingly, Annals Phys. 321 (2006) 150 arXiv:astro-ph/0505267.

[54] D. Baumann and D. Green, arXiv:1102.5343 [hep-th].

[55] A. J. Tolley and M. Wyman, Phys. Rev. D 81 (2010) 043502 [arXiv:0910.1853 [hep-th]]. 
[56] D. Babich, P. Creminelli and M. Zaldarriaga, JCAP 0408, 009 (2004) arXiv:astro-ph/0405356.

[57] A. Ashoorioon, D. Chialva, U. Danielsson, JCAP 1106 (2011) 034. [arXiv:1104.2338 [hep-th]].

[58] J. Martin and D. J. Schwarz, Phys. Rev. D 67 (2003) 083512 arXiv:astro-ph/0210090.

[59] C. M. Bender, S. A. Orszag, "Asymptotic Methods for Scientists and Engeneers", McGraw Hill, 1978, (Springer 1999).

[60] M. Porrati, Proceedings of 13th International Seminar on High-Energy Physics: Quarks 2004, Pushkinskie Gory, Russia, 24-30 May 2004 arXiv:hep-th/0409210.

[61] M. Porrati, Phys. Lett. B 596 (2004) 306 arXiv:hep-th/0402038.

[62] N. D. Birrell,P. C. W. Davies, "Quantum Fields in Curved Space" Cambridge University Press (1984-1994).

[63] E. Mottola, Phys. Rev. D 31 (1985) 754.

[64] L. Parker, Phys. Rev. Lett. 21 (1968) 562.

[65] L. Parker, Phys. Rev. 183 (1969) 1057.

[66] L. Parker, Phys. Rev. D 3 (1971) 346 [Erratum-ibid. D 3 (1971) 2546].

[67] S. A. Fulling, "Aspects of Quantum Field Theory in Curved Space-Time", Cambridge University Press, 1989

[68] P. Creminelli, JCAP 0310 (2003) 003 arXiv:astro-ph/0306122.

[69] A. Erdélyi, "Asymptotic Expansions", Dover (New York) 1956.

[70] C. Cheung, A. L. Fitzpatrick, J. Kaplan and L. Senatore, JCAP 0802 (2008) 021 arXiv:0709.0295 [hep-th]]. 